\title{
Market Complete Option Valuation using a Jarrow-Rudd Pricing Tree with Skewness and Kurtosis
}

\author{
Yuan $\mathrm{Hu}^{1}$, Abootaleb Shirvani ${ }^{1,3}$, W. Brent Lindquist, ${ }^{1,}$, Frank J. Fabozzi ${ }^{2}$, and Svetlozar \\ T. Rachev ${ }^{1}$ \\ ${ }^{1}$ Department of Mathematics \& Statistics, Texas Tech University, Lubbock, TX 79409-1042, USA, \\ yuan.hu@ttu.edu; abootaleb.shirvani@ttu.edu; zari.rachev@ttu.edu \\ ${ }^{2}$ Finance Department, EDHEC Business School, 393/400 Promenade des Anglais-BP3116, CEDEX 3, 06202 Nice, \\ France, frank.fabozzi@edhec.edu \\ ${ }^{3}$ Present address: Department of Actuarial Science \& Risk Management, Drake University, Des Moines, IA, 50311, \\ USA \\ ${ }^{*}$ Corresponding author: brent.lindquist@ttu.edu
}

June 18, 2021

\begin{abstract}
Applying the Cherny-Shiryaev-Yor invariance principle, we introduce a generalized Jarrow-Rudd (GJR) option pricing model with uncertainty driven by a skew random walk. The GJR pricing tree exhibits skewness and kurtosis in both the natural and risk-neutral world. We construct implied surfaces for the parameters determining the GJR tree. Motivated by Merton's pricing tree incorporating transaction costs, we extend the GJR pricing model to include a hedging cost. We demonstrate ways to fit the GJR pricing model to a market driver that influences the price dynamics of the underlying asset. We supplement our findings with numerical examples.
\end{abstract}

Keywords Jarrow-Rudd binomial option pricing; skew random walk; Cherny-Shiryaev-Yor invariance principle; hedging transaction cost 


\section{Introduction}

Pricing trees determined by a Markov chain have been studied in a number of academic papers 1 Since the Markov chain pricing tree is constructed directly in the risk-neutral world in these papers, it is not clear what discrete pricing model in the natural world would evolve to a risk-neutral Markov chain pricing model in accordance with dynamic asset pricing theory 2 In this work, we follow the classical binomial pricing model framework (see Cox et al. (1979) and Jarrow and Rudd (2012, Chapters V and VI)). We begin with a Markov chain model in the natural world replicating a self-financing portfolio, and then transform to risk-neutral option pricing preserving market completeness. It is essential that our option pricing model is defined first in the natural world. If the option pricing model is placed directly in the risk-neutral world, and calibrated with market option data, no option market dislocation or option mispricing can be revealed. Option pricing models which do not start with modeling the underlying assets in the natural world and then, via risk-neutral valuation based on Black-Scholes-Merton dynamic pricing theory, pass to the risk-neutral world are meaningless, if not dangerous, in practical applications. Such pricing models are generally used to predict what the option traders believe the correct option prices are and not what option prices are actually fair. It is imperative that opinion prices be aligned with reliable spot market models 3

To illustrate this claim, suppose that a trader would like to determine if potential mispricing in the option market exists due to a market bubble that the trader suspects will burst 4 The trader's option pricing model, when calibrated to option market data, should recover, uniquely, the spot price dynamics (Ross, 2015) of the underlying asset (Kim et al. (2016), Hu et al. (2020a)). If that recovered spot price process does not conform with market data on the asset spot price, this could be a trading signal that there is potential option mispricing 5

Our paper is close in spirit to Kijima and Yoshida (1993), where an option pricing model with Markov chain stochastic volatility is introduced and the continuous-time limiting price process is determined as a subordinated geometric Brownian motion (GBM) 6 While the discrete- and continuous-time market models are incomplete in the paper by Kijima and Yoshida, in our paper we deal with complete market models, both in the discrete- and continuous-time settings. We extend the Jarrow and Rudd (JR) binomial pricing model (Jarrow and Rudd (2012), Hull (2012, p. 442), Kim et al. $(2016,2019)$ ) with an additional parameter determining the skewness and excess kurtosis of the underlying asset return distribution. We refer to our extended model as the generalized Jarrow-Rudd (GJR) option pricing model.

As in the original JR model (Kim et al., 2016, 2019; Hu et al., 2020a,b), our GJR pricing model preserves market completeness 7 In the GJR pricing model, the embedded Markov chain driving the discrete underlying price process is a skew random walk which, in the limit, becomes a skew Brownian motion (SBM) after the necessary scalar normalization 8 In discrete time, the distributional mapping between the GJR price dynamics of the underlying asset and its risk-neutral dynamics is one-to-one 9 The GJR pricing model

\footnotetext{
${ }^{1}$ See, for example, Duan and Simonato (2001), Simonato (2011), Bhat and Kumar (2012), Fuh et al. (2012), Van and Elliot (2012a,b), and Fan et al. (2016). In Bhat and Kumar (2012), the authors claim that the limiting return distribution is a mixture of normal distributions. A mixture of different normal distributions is not infinitely divisible (Steutel and van Harn, 2004, Chapter VI), and it is therefore not clear that their limiting continuous-time model is arbitrage-free.

${ }^{2}$ Black and Scholes (1973), Merton (1973), Delbaen and Schachermaver (1994), Delbaen and Schachermaver (1998), and Duffie (2001, Chapter 6).

${ }^{3}$ Black (1975), Brenner and Galai (1984), Melick and Thomas (1997), Hilber et al. (2009), Pasquariello (2014), and Ross $(2015)$.

${ }^{4}$ The trader can decide to ride the bubble as long as possible, as many other option traders will do, with the hope of unwinding option positions before the bubble bursts. In this case, the trader does not need to worry about what the true spot market model is that corresponds to the risk-neutral option pricing model. For an extensive study of market bubbles and related option markets, we refer to Heston et al. (2007), Jarrow et al. (2010), and Vogel (2018).

${ }^{5}$ In this setting model risk does exist. That is why in real trading a suite of option pricing models is used.

${ }^{6}$ We employ the following abbreviations throughout this paper. Each abbreviation is also defined the first time it is referenced. BM: Brownian motion; CPM: continuous-time option pricing model; CSYIP: Cherny-Shiryaev-Yor Invariance Principle; DPM: discrete-time option pricing model; ECC: European contingency claim; GBM: geometric Brownian motion; GJR: generalized Jarrow-Rudd; HTC: hedging transaction cost; JR: Jarrow-Rudd; RMSE: relative mean-square error; SBM: skew Brownian motion; w.p.: with probability.

${ }^{7}$ As far the authors of this paper are aware, all discrete market models in the literature exhibiting skewness and kurtosis lead to incomplete market models. Thus, the problem of determining the unique spot price tree corresponding to the risk-neutral tree chosen in any of those papers will be unsolved.

${ }^{8}$ See Harrison and Shepp (1981), Cherny et al. (2003), Revuz and Yor (1994, Chapters VII and X), and Corns and Satchell (2007).

${ }^{9}$ That is, for every fixed trading frequency, the probability law of the risk-neutral tree for the underlying asset uniquely
} 
allows us to study option pricing in a Markov chain market model with transaction costs. Our market model has a relatively simple parametric form and is easily calibrated to option data, as illustrated by numerical examples.

Our paper proceeds as follows. In section 2 we introduce the Cherny-Shiryaev-Yor invariance principle (CSYIP) (Cherny et al., 2003) for SBM. As with the use of the Donsker-Prokhorov invariance principle10 in the Cox-Ross-Rubinstein pricing tree (Cox et al., 1979) and in the JR pricing tree, we apply CSYIP to introduce the GJR pricing tree and obtain the limiting continuous-time price dynamics. The mean return $\mu$ is retained as a parameter in the GJR model. The GJR tree includes an additional parameter $\beta$ governing the skewness and kurtosis of the GJR tree in the natural world. While the GJR tree ultimately converges to a GBM as in the classical JR pricing tree, the GJR pre-limiting behavior is that of geometric SBM. We investigate numerically the pre-limiting behavior of the GJR tree to obtain estimates of the smallest number, $n$, of trading intervals to maturity $T=n \Delta t, n \uparrow \infty$, at which the skewness and excess kurtosis vanish from the GJR tree distribution. In section 3 , we determine the risk-neutral probabilities in the GJR pricing model which, together with the volatility $\sigma$ and the risk-free rate $r_{f}$, depend on $\mu$ and $\beta$. Using daily closing price data for the SPDR S\&P 500 ETF Trust fund (SPY) and call option data for the same underlying asset, we estimate the implied $\mu, \beta$ and $\sigma$ surfaces. Motivated by Merton's binomial tree model with transaction costs (Merton, 1990, Chapter 14), in section 4 we extend the GJR pricing tree model to include a hedging transaction cost term. Using the SPY option data, we estimate option transaction costs, the implied transaction cost surface, and the impact transaction costs have on the implied $\mu, \beta$ and $\sigma$ surfaces. In section 5 , we explore possibilities for fitting the GJR model to a market driver that affects the price dynamics of the underlying asset. We estimate the parameters for the new pricing tree model in a numerical example with the underlying asset being the stock of Microsoft Corporation (MSFT). We explore both an endogenous and exogenous approach. The exogenous approach allows for greater generalization of the market driver; in a numerical example we assume the asset returns are dependent on Fama-French five-factor loading values (Fama and French, 2015). In section 6, we derive the risk-neutral probabilities for the extended pricing tree introduced in section 5 and estimate the corresponding implied volatility surface. Chapter 7 concludes of our work.

\section{The generalized Jarrow-Rudd pricing tree model}

In this section, we introduce the GJR pricing tree model. To do so, we describe SBM and then apply the CSYIP to formulate a new path-dependent pricing model defined by a recombined tree that generalizes the JR binomial option pricing model 11 In contrast to the classical Cox-Ross-Rubinstein and JR binomial pricing models, in the GJR pricing model the pre-limiting pricing process is determined by SBM. That is, for a moderately small trading frequency $\Delta t$, the GJR dynamics exhibit the properties of geometric SBM. This feature leads to a more flexible discrete pricing model for the underlying asset price behavior for any realistically small trading frequency. However, as $\Delta t \downarrow 0$ our pricing tree converges to a GBM, as in the traditional JR pricing model. As shown in $\mathrm{Hu}$ et al. (2020a,b), discrete-time option pricing models contain considerably more information than continuous-time pricing models.

Due to the option trader's presumed ability to trade continuously with no transaction costs, in a continuous-time option pricing model the information about the underlying stock mean return and stockprice direction at a given trading frequency is lost. That is the main reason that we emphasize a discrete-time option pricing model, rather than paying more attention to the limiting continuous-time option price dynamics. In the GJR option pricing model, new features, such as skewness and excess kurtosi 12 , of the underlying stock return distribution will be present.

determines the probability law of the asset's pricing tree in the natural world.

10 Donsker (1951), Prokhorov (1956), Billingslev (1999, section 14), Gikhman and Skorokhod (1969, Chapter IX), Skorokhod (2005, section 5.3.3), Davydov and Rotar (2008)

${ }^{11}$ See Jarrow and Rudd (2012); Hull (2012, p. 442); Kim et al. (2016, 2019).

${ }^{12}$ Discrete-time option pricing models with underlying stock return distributions exhibiting skewness and excess kurtosis are known; see, for example, Yamada and Primbs (2004). But the Yamada-Primbs' pricing model is based on mean-variance hedging in incomplete markets. Our GJR pricing model is based on no-arbitrage asset valuation arguments leading to a complete market model. 


\subsection{Definition and properties of SBM}

We start with the definition of a $\operatorname{SBM} 13$ Let $\mathbb{B}=\left\{B_{t}, t \geq 0\right\}$ be a standard Brownian motion (BM) generating a stochastic basis $\left(\Omega, \mathbb{F}=\left\{\mathcal{F}_{t}=\sigma\left(B_{u}, u \leq t\right)_{t \geq 0}\right\}, \mathbb{P}\right)$. Let $\alpha \in[0,1]$ and set 14

$$
\begin{aligned}
\mathcal{A}_{t}^{(\alpha)} & =\int_{0}^{t}\left(\alpha^{2} I_{\left\{B_{s} \geq 0\right\}}+(1-\alpha)^{2} I_{\left\{B_{s}<0\right\}}\right) d s, \\
\tau_{t}^{(\alpha)} & =\inf \left\{s \geq 0: \mathcal{A}_{s}^{(\alpha)}\right\}, \\
B_{t}^{(\alpha)} & =\varphi_{\alpha}\left(B_{\tau_{t}^{(\alpha)}}\right), t \geq 0,
\end{aligned}
$$

where $\varphi_{\alpha}(x)=\alpha x I_{\{x \geq 0\}}+(1-\alpha) x I_{\{x<0\}}, x \in R$, and $I_{\{\cdot\}}$ is the indicator function. The process $\mathbb{B}^{(\alpha)}=$ $\left\{B_{t}^{(\alpha)}, t \geq 0\right\}$ with $B_{0}^{(\alpha)}=0$ is a SBM with parameter $\alpha$ having the following properties 15

(i) $\mathbb{B}^{(1)} \stackrel{\mathrm{d}}{=}|\mathbb{B}|=\left\{\left|B_{t}\right|, t \geq 0\right\} \underline{16}$

(ii) $\mathbb{B}^{(1 / 2)} \stackrel{\mathrm{d}}{=}|\mathbb{B}|$.

(iii) $\mathbb{B}^{(0)} \stackrel{\mathrm{d}}{=}-|\mathbb{B}|$.

(iv) $\left|\mathbb{B}^{(\alpha)}\right| \stackrel{\mathrm{d}}{=}|\mathbb{B}|$.

(v) $\mathbb{B}^{(\alpha)}$ is a semimartingale satisfying the strong Markov property.

(vi) When $t \geq 0$, sample paths of $\mathbb{B}^{(\alpha)}$ can be generated using the representation 17

$$
B_{t}^{(\alpha)}=\left\{\begin{aligned}
\left|B_{t}\right|, & \text { w.p. } \alpha, \\
-\left|B_{t}\right|, & \text { w.p. } 1-\alpha .
\end{aligned}\right.
$$

(vii) For $0 \leq s<t$,

$$
\mathbb{P}\left(B_{s+t}^{(\alpha)} \in d x \mid B_{s}^{(\alpha)}=0\right)=\left\{\begin{array}{l}
\alpha \sqrt{2 / \pi t} \exp \left(-\frac{x^{2}}{2 t}\right) d x, \text { if } x \geq 0 \\
(1-\alpha) \sqrt{2 / \pi t} \exp \left(-\frac{x^{2}}{2 t}\right) d x, \text { if } x<0
\end{array}\right.
$$

is the conditional density $f_{t}^{(\alpha)}(x), x \in R$, with conditional cumulative distribution function $F_{t}^{(\alpha)}(x)=$ $\int_{-\infty}^{x}(y) d y, x \in R$, given by

$$
F_{x}^{(\alpha)}(x)=\left\{\begin{array}{l}
(1-\alpha)+2 \alpha / \sqrt{\pi} \int_{0}^{x / \sqrt{2 t}} \exp \left(-z^{2}\right) d z, \text { if } x \geq 0 \\
(1-\alpha)\left(1-2 / \sqrt{\pi} \int_{x / \sqrt{2 t}}^{0} \exp \left(-z^{2}\right) d z\right), \text { if } x<0
\end{array}\right.
$$

(viii) Corns and Satchell (2007) developed a continuous-time option pricing model based on geometric Azzalini SBM. The trajectories $A_{t}^{(\delta)}(\omega), t \geq 0, \omega \in \Omega$, of an Azzalini SBM, $\mathbb{A}^{(\delta)}=\left\{A_{t}^{(\delta)}, t \geq 0\right\}$, with parameter $\delta \in(-1,1)$ have the form $A_{t}^{(\delta)}(\omega)=\sqrt{1-\delta^{2}} B_{1, t}(\omega)+\delta\left|B_{2, t}(\omega)\right|$, where $B_{1, t}$ and $B_{2, t}$ are two independent BMs. Corns and Satchell (2007) showed that $\mathbb{A}^{(\delta)} \stackrel{\mathrm{d}}{=} \mathbb{B}^{(\alpha)}$ with $\alpha=(1+\delta) / 2$.

\footnotetext{
${ }^{13}$ See Itô and McKean (1996, section 4.2, problem 1, p. 115); Harrison and Shepp (1981); Revuz and Yor (1994, Chapters VII and X ); Lang (1995); Lejay (2006); Corns and Satchell (2007); Cherny et al. (2003); Ramirez (2011); Atar and Budhiraja (2015); Trutnau et al. (2015); and Li (2019).

${ }^{14}$ See Chernv et al. (2003).

${ }^{15}$ See Cherny et al. (2003, Chapter 4) and Corns and Satchell (2007).

${ }^{16}$ Here, and in what follows, $\stackrel{\mathrm{d}}{=}$ stands for "equal in distribution" or "equal in probability law".

${ }^{17}$ See Itô and McKean (1996, section 4.2, problem 1, p. 115), Leiav (2006) and Corns and Satchell (2007). We denote "with probability" as "w.p."
} 
(ix) The moment-generating function of $B_{t}^{(\alpha)}$ has the form

$$
M_{B_{t}^{(\alpha)}}(u)=\mathbb{E}\left(\exp \left(u B_{t}^{(\alpha)}\right)\right)=\exp \left(u^{2} t / 2\right)\left(1+(2 \alpha-1) \frac{2}{\sqrt{\pi}} \int_{0}^{u \sqrt{t / 2}} \exp \left(-z^{2}\right) d z\right), u>0
$$

The moments $\mu_{t}^{(p, \alpha)}=\mathbb{E}\left(\left(B_{t}^{(\alpha)}\right)^{p}\right)$ are given by

$$
\mu_{t}^{(p, \alpha)}=\sqrt{\frac{2^{p}}{\pi}} \Gamma\left(\frac{p+1}{2}\right)\left(\alpha+(-1)^{p}(1-\alpha)\right) t^{p / 2}, p>0, s \geq 0, t>0 .
$$

Note that odd moments depend on $\alpha$ through the term $2 \alpha-1$, while even moments are independent of $\alpha$.

From (2), the mean, variance, skewness and excess kurtosis of $\mathbb{B}^{(\alpha)}$ are given by

$$
\begin{aligned}
\mu_{t}^{(\alpha)} & =\mu_{t}^{(1, \alpha)}=(2 \alpha-1) \sqrt{2 t / \pi} \\
V_{t}^{(\alpha)} & =\mu_{t}^{(2, \alpha)}-\left(\mu_{t}^{(\alpha)}\right)^{2}=\left(1-2(2 \alpha-1)^{2} / \pi\right) t \\
\gamma^{(\alpha)} & =\frac{\mathbb{E}\left(\left(B_{t}^{(\alpha)}-\mu_{t}^{(\alpha)}\right)^{3}\right)}{\left(V_{t}^{(\alpha)}\right)^{3 / 2}}=\frac{\sqrt{2}(2 \alpha-1)\left(4(2 \alpha-1)^{2}-\pi\right)}{\left(\pi-2(2 \alpha-1)^{2}\right)^{3 / 2}}, \\
\nu^{(\alpha)} & =\frac{\mathbb{E}\left(\left(B_{t}^{(\alpha)}-\mu_{t}^{(\alpha)}\right)^{4}\right)}{\left(V_{t}^{(\alpha)}\right)^{2}}-3=\frac{8 \pi(2 \alpha-1)^{2}-24(2 \alpha-1)^{4}}{\left(\pi-2(2 \alpha-1)^{2}\right)^{2}} .
\end{aligned}
$$

Note that the skewness and excess kurtosis are time-independent quantities. The behavior of these four quantities is demonstrated in Fig. 1.

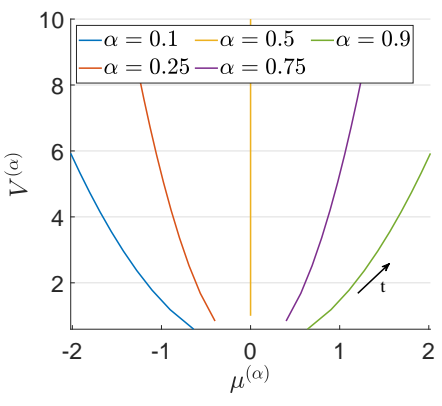

(a)

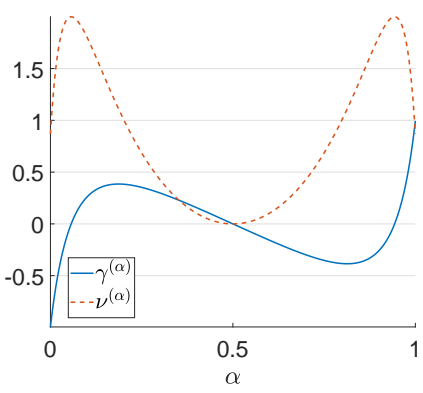

(b)

Figure 1: (a) Bivariate plot of mean $\mu_{t}^{(\alpha)}$ and variance $V_{t}^{(\alpha)}$ of a SBM under different parameter values of $\alpha$ and $t \in[0,10]$. (b) Plots of skewness $\gamma^{(\alpha)}$ and excess kurtosis $\nu^{(\alpha)}$ of a SBM as a function of $\alpha \in[0,1]$.

\subsection{CSYIP for SBM}

We start with the formulation of the CSYIP for SBM with the definition of a piecewise continuous function. A function $h: R \rightarrow R$ is called a piecewise continuous function if there exists a collection of disjoint intervals $J_{n}, n \in \mathcal{N}$, such that 18

\footnotetext{
${ }^{18}$ Each $J_{n}$ can be closed, open, semi-open, or a singleton. Define $\mathcal{N}=\{1,2, \ldots\}$ and $\mathcal{N}_{0}=\{0,1, \ldots\}$.
} 
$\operatorname{PSC}(\mathrm{i}) \quad \cup_{n=1}^{\infty} J_{n}=R$;

PSC(ii) for every compact interval $J$ there exists $n \in \mathcal{N}$ such that $\cup_{k=1}^{n} J_{k} \supseteq J$; and

$\operatorname{PSC}\left(\right.$ iii) on each $J_{n}, n \in \mathcal{N}, h: J_{n} \rightarrow R$ is continuous and has finite limits at those endpoints of $J_{n}$ which do not belong to $J_{n}$.

Next, for $\alpha \in(0,1)$, let $\mathbb{M}^{(\alpha)}=\left\{M_{k}^{(\alpha)} \in \mathbb{Z}, k \in \mathcal{N}_{0}\right\}, \mathbb{Z}=\{0, \pm 1, \pm 2, \ldots\}$ be a Markov chain with $M_{0}^{(\alpha)}=0$ and transition probabilitie: 19

$$
\begin{aligned}
& \mathbb{P}\left(M_{k+1}^{(\alpha)}=i+1 \mid M_{k}^{(\alpha)}=i\right)=\left\{\begin{array}{l}
\frac{1}{2}, \text { if } i \neq 0, \\
\alpha, \text { if } i=0,
\end{array}\right. \\
& \mathbb{P}\left(M_{k+1}^{(\alpha)}=i-1 \mid M_{k}^{(\alpha)}=i\right)=\left\{\begin{array}{l}
\frac{1}{2}, \text { if } i \neq 0, \\
1-\alpha, \text { if } i=0 .
\end{array}\right.
\end{aligned}
$$

We call $\mathbb{M}^{(\alpha)}$ a skew random walk with parameter $\alpha$. Fig. 2 shows the skew random walk for different values of $\alpha$. The results exhibit a predominance of trajectories having $M_{k}^{(\alpha)}<0$ for $\alpha<1 / 2$ and a predominance of trajectories having $M_{k}^{(\alpha)}>0$ for $\alpha>1 / 2$.

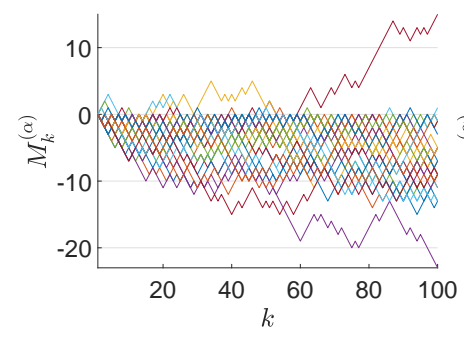

(a) $\alpha=0.1$

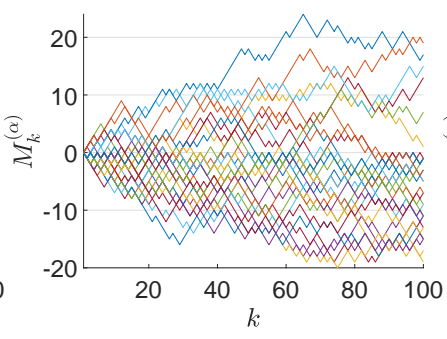

(b) $\alpha=0.25$

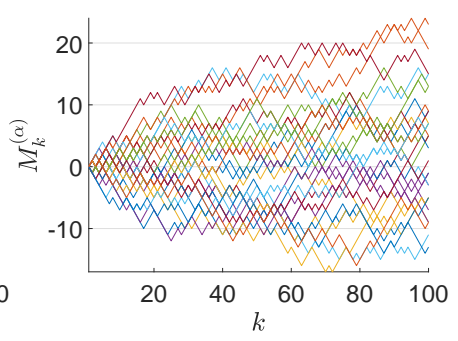

(c) $\alpha=0.5$

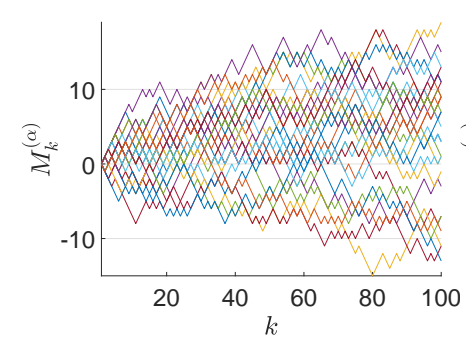

(d) $\alpha=0.75$

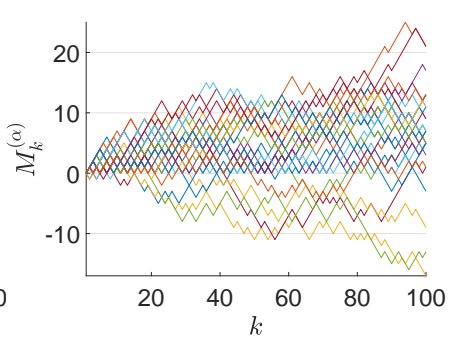

(e) $\alpha=0.9$

Figure 2: 30 Monte Carlo scenarios for $\mathbb{M}^{(\alpha)}=\left\{M_{k}^{(\alpha)} \in \mathbb{Z}, k=1, \ldots, 100\right\}$ for select values of $\alpha \in(0,1)$.

For $n \in \mathcal{N}$, set $M_{0}^{(\alpha, n)}=0, M_{k}^{(\alpha, n)}=n^{-1 / 2} M_{k}^{(\alpha)}$ and $X_{k / n}^{(\alpha, n)}=\sum_{i=1}^{k} M_{i}^{(\alpha, n)}, k=1, \ldots, n$. Fix $n \in \mathcal{N}$ and define $\mathbb{B}_{t}^{(\alpha, n)}, t \geq 0$, to be the random process with piecewise linear trajectories having vertexes $\left(k / n, \mathbb{B}_{k / n}^{(\alpha, n)}\right)$, where $\mathbb{B}_{k / n}^{(\alpha, n)}=X_{k / n}^{(\alpha, n)}$. Let $h: R \rightarrow R$ be a piecewise continuous function and define $Y_{k / n}^{(\alpha, n)}=\sum_{i=1}^{k} h\left(X_{(i-1) / n}^{(\alpha, n)}\right)\left(X_{i / n}^{(\alpha, n)}-X_{(i-1) / n}^{(\alpha, n)}\right), k=1, \ldots, n$. Define $\mathbb{C}_{t}^{(\alpha, n)}, t \geq 0$, to be a random process with the piecewise linear trajectories having vertexes $\left(k / n, \mathbb{C}_{k / n}^{(\alpha, n)}\right)$, where $\mathbb{C}_{k / n}^{(\alpha, n)}=Y_{k / n}^{(\alpha, n)}$.

CSYIP for a SBM 20: If $h: R \rightarrow R$ is a piecewise continuous function, then for $t \geq 0$, as $n \uparrow \infty$, the bivariate process $\left(\mathbb{B}_{t}^{(\alpha, n)}, \mathbb{C}_{t}^{(\alpha, n)}\right)$ converges in law to $\left(B_{t}^{(\alpha)}, C_{t}^{(\alpha)}\right)$, where $B_{t}^{(\alpha)}$ is a $S B M$ and $C_{t}^{(\alpha)}=$ $\int_{0}^{t} h\left(B_{s}^{(\alpha)}\right) d B_{s}^{(\alpha)}$.

\footnotetext{
${ }^{19}$ See Harrison and Shepp $(\sqrt{1981})$ and Cherny et al. $(2003)$.

${ }^{20}$ See Theorem 4.1 in Cherny et al. (2003).
} 


\subsection{The GJR pricing model}

We begin the construction of a binomial pricing tree by applying CSYIP using only the lower moment SBM process $\mathbb{B}_{t}^{(\alpha, n)}$. We will add the higher moment $\mathbb{C}_{t}^{(\alpha, n)}$ in section 5.2. We fix $n \in \mathcal{N}$, the time interval $\Delta t=T / n$, and $\beta \in(-1 / \sqrt{\Delta t}, 1 / \sqrt{\Delta t})$. Set

$$
\alpha_{\Delta t}=(1+\beta \sqrt{\Delta t}) / 2 \in(0,1) .
$$

If we consider the process $\mathbb{D}^{\left(\alpha_{\Delta t}\right)}=\left\{D_{t}=B_{k \Delta t}^{\left(\alpha_{\Delta t}\right)}, k \Delta t \leq t<(k+1) \Delta t, k=1, \ldots, n-1, D_{T}=B_{T}^{\left(\alpha_{\Delta t}\right)}\right\}$, then $\mathbb{D}^{\left(\alpha_{\Delta t}\right)}$ weakly converges in the Skorokhod space $\mathcal{D}[0, T]$ to the BM $\mathbb{B}$ as $\Delta t \downarrow 0$. However, for fixed $\Delta t, \mathbb{D}^{\left(\alpha_{\Delta t}\right)}$ exhibits the properties of a SBM with parameter $\alpha_{\Delta t}$. From (8), (77) and Fig. 2, we see that $\beta$ will affect the moments of the process $B_{k \Delta t}^{\left(\alpha_{\Delta t}\right)}$. This becomes clear by observing the form of the mean, variance, skewness and excess kurtosis of $B_{k \Delta t}^{(\alpha \Delta t)}$. From (3) - (6) , to leading order in $\Delta t, \mu_{k \Delta t}^{(\alpha \Delta t)}=\beta \sqrt{2 k / \pi} \Delta t$, $V_{k \Delta t}^{(\alpha \Delta t)}=k \Delta t, \gamma_{k \Delta t}^{(\alpha \Delta t)}=-\sqrt{2 \Delta t / \pi} \beta$, and $\nu_{k \Delta t}^{(\alpha \Delta t)}=8 \beta^{2} \Delta t / \pi$. Thus, for any fixed (arbitrarily small) time interval $\Delta t$, the increments $B_{k \Delta t}^{\left(\alpha_{\Delta t}\right)}$ have skewed and heavy tailed distributions when $\beta \neq 0$. Consider the arithmetic SBM

$$
B_{t}^{\left(\alpha ; \mu^{(\alpha)}, \sigma^{(\alpha)}\right)}=\mu^{(\alpha)} t+\sigma^{(\alpha)} B_{t}^{(\alpha)}, t \in[0, T],
$$

with mean $\mathbb{E}\left(B_{t}^{\left(\alpha ; \mu^{(\alpha)}, \sigma^{(\alpha)}\right)}\right)$ and variance $\operatorname{Var}\left(B_{t}^{\left(\alpha ; \mu^{(\alpha)}, \sigma^{(\alpha)}\right)}\right)$. Then, for $k=1, \ldots, n$, we have21

$$
\begin{aligned}
\mathbb{E}\left(B_{k \Delta t}^{\left(\alpha ; \mu^{(\alpha)}, \sigma^{(\alpha)}\right)}\right) & =\mu^{(\alpha)} k \Delta t+\sigma^{(\alpha)} \beta \sqrt{2 k / \pi} \Delta t, \\
\operatorname{Var}\left(B_{k \Delta t}^{\left(\alpha ; \mu^{(\alpha)}, \sigma^{(\alpha)}\right)}\right) & =\sigma^{(\alpha)^{2}} k \Delta t .
\end{aligned}
$$

We next define the GJR pricing tree, determined by the skew random walk $\mathbb{M}^{\left(\alpha_{\Delta t}\right)} 22$

GJR Pricing Tree: Let $\mu>0$ and $\sigma>0$. For $k=1, \ldots, n, n \Delta t=T, \alpha_{\Delta t}=(1+\beta \sqrt{\Delta t}) / 2$ and skew random walk $\mathbb{M}^{\left(\alpha_{\Delta t}\right)}$, define the GJR pricing recombined tree by

$$
S_{k \Delta t}^{(n)}=S_{0} \exp \left(v_{k} \Delta t+M_{k}^{\left(\alpha_{\Delta t}\right)} \sigma \sqrt{\Delta t}\right), k=1, \ldots, n,
$$

where $v_{k}=k \mu+\sigma \beta(\sqrt{2 k / \pi}-1)$. We study the limiting behavior of this tree as $\Delta t \downarrow 0$. Note that, to leading order in $\Delta t$,

$$
\mathbb{E}\left(M_{k}^{\left(\alpha_{\Delta t}\right)}\right)=2 \alpha-1=\beta \sqrt{\Delta t}, \quad \operatorname{Var}\left(M_{k}^{\left(\alpha_{\Delta t}\right)}\right)=k .
$$

Consider the cumulative log-return $R_{k \Delta t}^{(n)}=\ln \left(S_{k \Delta t}^{(n)} / S_{0}\right), k=1, \ldots, n$. From (10) and (11) we obtain

$$
\begin{aligned}
\mathbb{E}\left(R_{k \Delta t}^{(n)}\right) & =\mu k \Delta t+\sigma \beta \sqrt{2 k / \pi} \Delta t=\mu k \Delta t+\sigma \mathbb{E}\left(B_{k \Delta t}^{\left(\alpha_{\Delta t}\right)}\right), \\
\operatorname{Var}\left(R_{k \Delta t}^{(n)}\right) & =\sigma^{2} k \Delta t=\sigma^{2} \operatorname{Var}\left(B_{k \Delta t}^{\left(\alpha_{\Delta t}\right)}\right) .
\end{aligned}
$$

From (12) and the CSYIP it follows that, for a fixed but relatively small time-increment $\Delta t$, the pricing tree (10) approximates

$$
S_{t}=S_{0} \exp \left(\mu t+\sigma B_{t}^{\left(\alpha_{\Delta t}\right)}\right), S_{0}>0, t \in[0, T], \mu>0, \sigma>0 .
$$

However, if we let $\Delta t \downarrow 0$, then the $\mathcal{D}[0, T]$-process generated by the tree (10) will ultimately converge to a GBM, that is, $S_{t}=S_{0} \exp \left(\mu t+\sigma B_{t}\right), t \in[0, T]$.

${ }^{21}$ As $\Delta t=1 / n$, we can assume that the value of $\left[\mathbb{E}\left(B_{k \Delta t}^{\left(\alpha ; \mu^{(\alpha)}, \sigma^{(\alpha)}\right)}\right)\right]^{2}$ is negligible. Then

$$
\operatorname{Var}\left(B_{k \Delta t}^{\left(\alpha ; \mu^{(\alpha)}, \sigma^{(\alpha)}\right)}\right)=\mathbb{E}\left[\left(B_{k \Delta t}^{\left(\alpha ; \mu^{(\alpha)}, \sigma^{(\alpha)}\right)}\right)^{2}\right]-\left[\mathbb{E}\left(B_{k \Delta t}^{\left(\alpha ; \mu^{(\alpha)}, \sigma^{(\alpha)}\right)}\right)\right]^{2}=\sigma^{(\alpha)^{2}} k \Delta t
$$

\footnotetext{
${ }^{22}$ If $\alpha_{\Delta t}=1 / 2$ (i.e. $\beta=0$ ), we obtain the JR binomial tree. Jarrow and Rudd $(2012)$ and Hull (2012, p. 442) defined the JR binomial tree directly in the risk-neutral world, that is, when $\mu=r_{f}$, where $r_{f}$ is the risk-free rate. Kim et al. (2016). showed that JR binomial tree option pricing model provides the fastest rate of convergence to the corresponding GBM in the risk-neutral world.
} 


\subsection{An example}

As a numerical example to investigate the pre-limiting behavior of the GJR pricing tree, we use SPY daily closing prices. Price data covering $N=7136$ trading days over the period $1 / 29 / 1993$ to $6 / 1 / 2021$ was obtained from Bloomberg Professional Services. We denote SPY daily closing prices as $S_{k \Delta t}^{(\mathrm{SPY})}$ and cumulative log-returns as $R_{k \Delta t}^{(\mathrm{SPY})}=\ln \left(S_{k \Delta t}^{(\mathrm{SPY})} / S_{0}^{(\mathrm{SPY})}\right)$, where $k=1, \ldots, N$, and $\Delta t=1 / 252$. We first determine a length, $L$, for a moving-window estimator that provides relatively strong explanatory power and acceptable precision for computing the parameters $\mu, \sigma$ and $\beta$, by using the following procedure based on equations (12). For each tested length $L$ and for each moving window $w_{i}, i=1, \ldots, N-L+1$, repeat the following steps.

Step 1: Fit a robust linear regression using a logistic weight function 23 to the SPY return data in $w_{i}$ using the mode 24

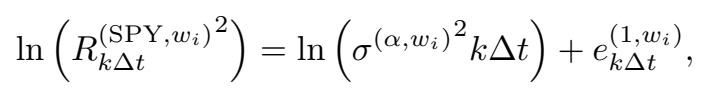

to produce estimates for the value of $\hat{\sigma}^{\left(\alpha, w_{i}\right)}$ and the error terms $e_{k \Delta t}^{\left(1, w_{i}\right)}, k=1, \ldots, L$.

Step 2: With $\hat{\sigma}^{\left(\alpha, w_{i}\right)}$ estimated from Step 1, apply the conditional least squares optimization,

$$
\begin{aligned}
& \min _{\beta^{\left(\alpha, w_{i}\right)} \in(-1 / \sqrt{\Delta t}, 1 / \sqrt{\Delta t})}\left\|e_{k \Delta t}^{\left(2, w_{i}\right)}\right\|_{2}^{2} \\
& =\min _{\beta^{\left(\alpha, w_{i}\right)} \in(-1 / \sqrt{\Delta t}, 1 / \sqrt{\Delta t})}\left\|\mathbb{E}\left(R_{k \Delta t}^{\left(\mathrm{SPY}, w_{i}\right)}\right)-\mu^{\left(\alpha, w_{i}\right)} k \Delta t-\hat{\sigma}^{\left(\alpha, w_{i}\right)} \beta^{\left(\alpha, w_{i}\right)} \sqrt{2 k / \pi} \Delta t\right\|_{2}^{2},
\end{aligned}
$$

to determine $\hat{\beta}^{\left(\alpha, w_{i}\right)}, \hat{\mu}^{\left(\alpha, w_{i}\right)}$ and the second error sequence $e_{k \Delta t}^{\left(2, w_{i}\right)}, k=1, \ldots, L$.

Step 3: Define e to be the random variable having the sample

$$
e_{k \Delta t}^{\left(w_{i}\right)}=\frac{e_{k \Delta t}^{\left(1, w_{i}\right)}+e_{k \Delta t}^{\left(2, w_{i}\right)}}{\sqrt{\left(e_{k \Delta t}^{\left(1, w_{i}\right)}\right)^{2}+\left(e_{k \Delta t}^{\left(2, w_{i}\right)}\right)^{2}}}, k=1, \ldots, L,
$$

and compute the $p$-value of the two-sided z-test with the hypothesis $H_{0}: \mathbb{e}=0$.

The statistics of the $p$-values computed in Step 3 using tested values of $L$ ranging from one month to four years are shown as box-whisker plots in Fig. 3. The $p$-value ranges indicate stronger rejection of $H_{0}$ as $L$ increases. As a compromise between satisfying the null hypothesis and retaining the accuracy provided by larger values of $L$, we chose $L=$ one year.

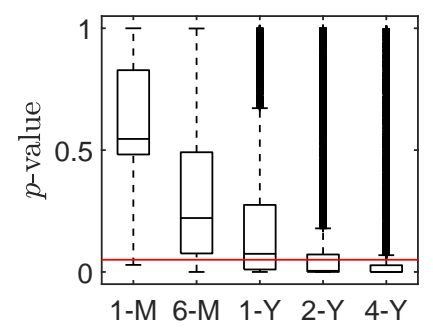

Figure 3: Box-whisker plot of the $p$-values for two-sided z-tests for different window lengths $L$. The horizontal line indicates the 0.05 significance level for rejecting the null hypothesis $H_{0}: \mathbb{e}=0$.

${ }^{23}$ See Holland and Welsch (2007) and Pregibon (1981).

${ }^{24}$ Since the cumulative return is not stationary, we employ the model $\operatorname{Var}\left(R_{k \Delta t}^{(\mathrm{SPY})}\right) \propto\left(R_{k \Delta t}^{(\mathrm{SPY})}\right)^{2}$. 


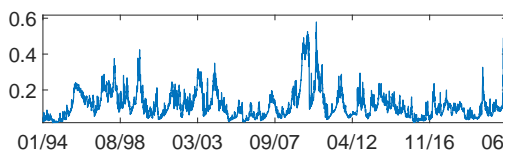

(a) $\hat{\sigma}^{(\alpha)}$

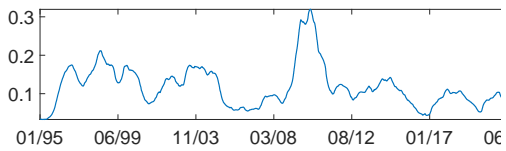

(d) $\bar{\sigma}^{(\alpha)}$

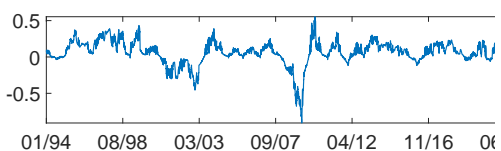

(b) $\hat{\mu}^{(\alpha)}$

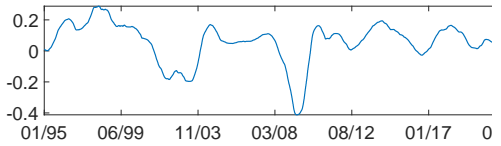

(e) $\bar{\mu}^{(\alpha)}$

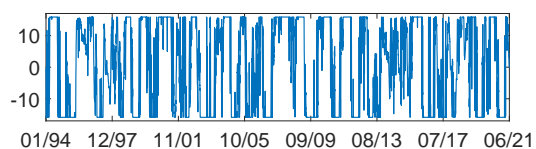

(c) $\hat{\beta}^{(\alpha)}$

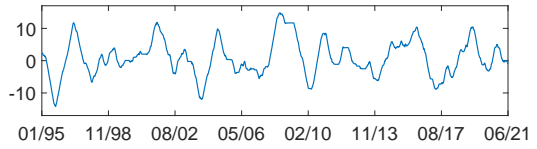

(f) $\bar{\beta}^{(\alpha)}$

Figure 4: Time series of the values (a) $\hat{\sigma}^{(\alpha)}$ estimated from Step 1, and (b) $\hat{\mu}^{(\alpha)}$ and (c) $\hat{\beta}^{(\alpha)}$ estimated from Step 2 using the $L=$ one year moving window. Plots (d) to (f) show 1-year moving average values of (a) to (c), repectively.

Fig. 4 presents the results for $\hat{\mu}^{(\alpha)}, \hat{\sigma}^{(\alpha)}$ and $\hat{\beta}^{(\alpha)}$ computed from the one-year moving window. The results clearly show a sharp drop in $\hat{\mu}^{(\alpha)}$ and increased volatility $\left(\hat{\sigma}^{(\alpha)}\right)$ resulting from the 2008 global financial crisis. The most recent peak in $\hat{\sigma}^{(\alpha)}$ in 2020 corresponds to the market reaction to the Covid-19 pandemic. In Fig. 4c, we note that optimum values for $\hat{\beta}^{(\alpha)}$ change rapidly and frequently hit the limits $\pm 1 / \sqrt{\Delta t}$. These results suggest that the least squares optimization in Step 2 may be improved with a smaller value of $\Delta t$, which would require intra-day data. We therefore smooth the time-series data in Figs. 4a to $4 \mathrm{c}$ using one-year moving averages. The time-series for the smoothed parameters, denoted $\bar{\sigma}^{(\alpha)}, \bar{\mu}^{(\alpha)}$ and $\bar{\beta}^{(\alpha)}$, are presented in Figs. 4d to 4f. The impact of the global financial crises is retained in the smoothed series. As the market disruption due to the Covid-19 pandemic was of shorter duration, the pandemic impact is lessened in the averaged data. Most significantly, the averaged values $\bar{\beta}^{(\alpha)}$ are better behaved.

Using the last one-year estimation window (from $6 / 2 / 2020$ to $6 / 1 / 2021$ ), we obtain the estimates $\bar{\sigma}^{(\alpha)}=$ $0.151, \bar{\mu}^{(\alpha)}=0.119, \bar{\beta}^{(\alpha)}=-0.978$, and $\bar{\alpha}=\left(1+\bar{\beta}^{(\alpha)} \sqrt{\Delta t}\right) / 2=0.469$ for the date $6 / 1 / 2021$. Based on these estimates, Fig. 5 shows a constructed, recombined, GJR price tree comprised of 30 price trajectories (10).

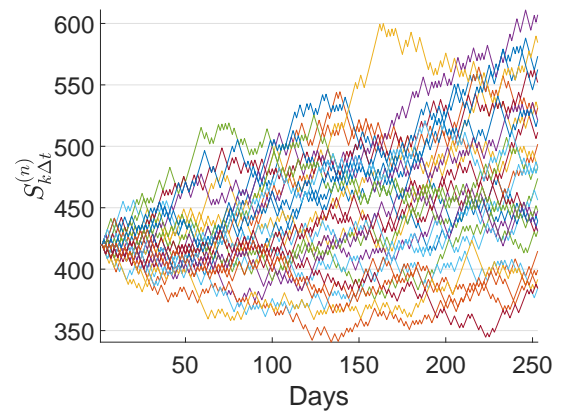

Figure 5: A recombined, GJR price tree (10) computed from the smoothed parameter estimates $\bar{\sigma}^{(\alpha)}=$ $0.151, \bar{\mu}^{(\alpha)}=0.119, \bar{\beta}^{(\alpha)}=-0.978$, and $\bar{\alpha}=0.469$. 30 price trajectories are displayed with initial capital $\$ S_{0}=419.67$, which corresponds to the SPY closing price on $6 / 1 / 2021$. 


\section{Option pricing using the GJR pricing tree}

We now consider the following discrete version of Corns' and Satchell's extension 25 of the Black-ScholesMerton market model 26 Suppose the dynamic:27 of the risky asset follows the GJR pricing tree (10). The dynamic 28 of the riskless asset is given by

$$
\beta_{k \Delta t}^{(n)}=\beta_{0} \exp \left(r_{f} k \Delta t\right), \beta_{0}>0, k=0,1, \ldots, n, \mu>r_{f}>0 .
$$

Let

$$
f_{k \Delta t}^{(n)}=f\left(S_{k \Delta t}^{(\mathcal{S})}, k \Delta t\right), k=1, \ldots, n,
$$

where $S_{k \Delta t}^{(\mathcal{S})}$ is the spot price of the underlying asset, be the price dynamics of a European Contingent Claim (ECC 29 having terminal time $n \Delta t=T>0$ and final payoff $f_{T}^{(n)}$. Following the construction of the CoxRoss-Rubinstein and JR binomial pricing models, our next goal is to use the pricing tree (10) to derive the price dynamics of $f_{k \Delta t}^{(n)}, k=0,1, \ldots, n$.

We start by forming the replicating risk-neutral portfolio $P_{k \Delta t}^{(n)}=D_{k \Delta t}^{(n)} S_{k \Delta t}^{(n)}-f_{k \Delta t}^{(n)}, k=0,1, \ldots, n-1$, adapted to the filtration $\mathbb{F}^{\left(n, \alpha_{\Delta t}\right)}=\left\{\mathcal{F}_{k \Delta t}=\sigma\left(M_{1}^{\left(\alpha_{\Delta t}\right)}, \ldots, M_{k}^{\left(\alpha_{\Delta t}\right)}\right) k=1, \ldots, n, \mathcal{F}_{0}=\{\varnothing, \Omega\}\right\}$. Then, conditionally on $\mathcal{F}_{k \Delta t}$, the replicating portfolio should be riskless, that is $P_{(k+1) \Delta t}^{(n)}=D_{k \Delta t}^{(n)} S_{(k+1) \Delta t}^{(n)}-f_{(k+1) \Delta t}^{(n)}$. From (7) and (10), and defining 30

$$
\begin{aligned}
& S_{(k+1) \Delta t}^{(n, u)}=S_{0} \exp \left(v_{k+1} \Delta t+\left(M_{k}^{\left(\alpha_{\Delta t}\right)}+1\right) \sigma \sqrt{\Delta t}\right), \\
& S_{(k+1) \Delta t}^{(n, d)}=S_{0} \exp \left(v_{k+1} \Delta t+\left(M_{k}^{\left(\alpha_{\Delta t}\right)}-1\right) \sigma \sqrt{\Delta t}\right),
\end{aligned}
$$

conditionally on $\mathcal{F}_{k \Delta t}$, by (12) it follows that

$$
\begin{aligned}
& S_{(k+1) \Delta t}^{(n, u)}=S_{k \Delta t}^{(n)} \exp (\mu \Delta t+\sqrt{2 / \pi} \sigma \beta(\sqrt{k+1}-\sqrt{k}) \Delta t+\sigma \sqrt{\Delta t}), \\
& S_{(k+1) \Delta t}^{(n, d)}=S_{k \Delta t}^{(n)} \exp (\mu \Delta t+\sqrt{2 / \pi} \sigma \beta(\sqrt{k+1}-\sqrt{k}) \Delta t-\sigma \sqrt{\Delta t}) .
\end{aligned}
$$

The risk-neutrality assumption implies that $D_{k \Delta t}^{(n)} S_{(k+1) \Delta t}^{(n, u)}-f_{(k+1) \Delta t}^{(n, u)}=D_{k \Delta t}^{(n)} S_{(k+1) \Delta t}^{(n, d)}-f_{(k+1) \Delta t}^{(n, d)}$, and thus

$$
D_{k \Delta t}^{(n)}=\frac{f_{(k+1) \Delta t}^{(n, u)}-f_{(k+1) \Delta t}^{(n, d)}}{S_{k \Delta t}^{(n)} \exp (\mu \Delta t+\sqrt{2 / \pi} \sigma \beta(\sqrt{k+1}-\sqrt{k}) \Delta t)\left(e^{\sigma \sqrt{\Delta t}}-e^{-\sigma \sqrt{\Delta t}}\right)} .
$$

Furthermore, given $\mathcal{F}_{k \Delta t}$, the portfolio $P_{(k+1) \Delta t}^{(n)}=P_{(k+1) \Delta t}^{(n, u)}$ is riskless, leading to

$$
f_{k \Delta t}^{(n, u)}=D_{k \Delta t}^{(n)} S_{k \Delta t}^{(n, u)}-e^{-r_{f} \Delta t} P_{(k+1) \Delta t}^{(n, u)}=e^{-r_{f} \Delta t}\left(q_{n, k+1} f_{(k+1) \Delta t}^{(n, u)}+\left(1-q_{n, k+1}\right) f_{(k+1) \Delta t}^{(n, d)}\right),
$$

where the risk-neural probability $q_{n, k+1}$ for the time period $[k \Delta t,(k+1) \Delta t)$ is given by

$$
q_{n, k+1}=\frac{\exp \left(r_{f} \Delta t-\mu \Delta t-\sqrt{2 / \pi} \sigma \beta(\sqrt{k+1}-\sqrt{k}) \Delta t\right)-e^{\sigma \sqrt{\Delta t}}}{e^{\sigma \sqrt{\Delta t}}-e^{-\sigma \sqrt{\Delta t}}} .
$$

\footnotetext{
${ }^{25}$ See Corns and Satchell (2007, section 3) where the asset dynamics was determined by an Azzalini SBM, $\mathbb{A}^{(\delta)}$. In what follows we will use $\mathbb{B}^{(\alpha)}=\left\{B_{t}^{(\alpha)}, t \geq 0\right\}$ instead of $\left.\mathbb{A}^{(\delta)}\right)$. Constructing a binomial model using $\mathbb{A}^{(\delta)}$ instead of $\mathbb{B}^{(\delta)}$ would require two independent pricing trees to model the discrete price dynamics of the underlying asset, which is not desirable for derivative hedging.

${ }^{26}$ For the Black-Scholes-Merton market model, see Black and Scholes (1973) and Duffie (2001, Chapter 6).

${ }^{27}$ We call the risky asset a stock, and it will be denoted by $\mathcal{S}$.

${ }^{28}$ We call the riskless asset a bond, and it will be denoted by $\mathcal{B}$.

${ }^{29}$ We use the terms ECC, option, and derivative interchangeably.

${ }^{30}$ Here, "u" stands for upward movement and "d" for downward movement in the binomial tree.
} 
To leading term in $\Delta t, q_{n, k+1}$ has the form,

$$
q_{n, k+1}=(1-\theta \sqrt{\Delta t}+\beta \sqrt{2 \Delta t / \pi}(\sqrt{k+1}-\sqrt{k})) / 2,
$$

where $\theta=\left(\mu-r_{f}+\sigma^{2} / 2\right) / \sigma$ is the market price of risk. For $\beta=0$, we obtain the risk-neutral probabilities under the JR binomial model (see Kim et al. (2016, 2019)).

Now we consider the risk-neutral dynamics of the stock $\mathcal{S}$ under the GJR pricing tree model (10) and the ECC dynamics (17). Given $\mathcal{F}_{k \Delta t}$, to leading order in $\Delta t$,

$$
S_{(k+1) \Delta t}^{(n ; q)}=\left\{\begin{array}{l}
S_{(k+1) \Delta t}^{(n, u ; q)}=S_{k \Delta t}^{(n)} \exp (\mu \Delta t+\sqrt{2 / \pi} \sigma \beta(\sqrt{k+1}-\sqrt{k}) \Delta t+\sigma \sqrt{\Delta t}), \text { w.p. } q_{n, k+1}, \\
S_{(k+1) \Delta t}^{(n, d)}=S_{k \Delta t}^{(n)} \exp (\mu \Delta t+\sqrt{2 / \pi} \sigma \beta(\sqrt{k+1}-\sqrt{k}) \Delta t-\sigma \sqrt{\Delta t}), \text { w.p. } 1-q_{n, k+1},
\end{array}\right.
$$

where $k=1, \ldots, n-1$. Conditionally on $\mathcal{F}_{k \Delta t}$, the discrete risk-neutral return $R_{(k+1) \Delta t}^{(n ; q)}=\ln \left(S_{(k+1) \Delta t}^{(n ; q)} / S_{k \Delta t}^{(n ; q)}\right)$, has mean $\mathbb{E}\left(R_{(k+1) \Delta t}^{(n ; q)}\right)=\left(r_{f}-\sigma^{2} / 2\right) \Delta t$ and variance $\operatorname{Var}\left(R_{(k+1) \Delta t}^{(n ; q)}\right)=\sigma^{2} \Delta t$, and for $\gamma>2, \mathbb{E}\left(\left|R_{(k+1) \Delta t}^{(n ; q)}\right|^{\gamma}\right)=$ $o(\Delta t)=0$. Let

$$
\begin{aligned}
& \mathbb{S}_{[0, T]}^{(n ; q)}=\left\{S_{t}^{(n ; q)}=S_{(k \Delta t)}^{(n ; q)}, t \in[k \Delta t,(k+1) \Delta t), k=0, \ldots, n-1, S_{T}^{(n ; q)}=S_{n \Delta t}^{(n ; q)}\right\}, \\
& \mathbb{S}_{[0, T]}^{(q)}=\left\{S_{0} \exp \left(\left(r_{f}-\sigma^{2} / 2\right) t+\sigma B_{t}^{(q)}\right), t \in[0, T]\right\},
\end{aligned}
$$

where $\mathbb{B}_{[0, T]}^{(q)}=\left\{B_{t}^{(q)}, t \in[0, T]\right\}$ is a standard BM. Then, by the Donsker-Prokhorov invariance principle, it follows that $\mathbb{S}_{[0, T]}^{(n, q)}$ converges weakly in $\left(\mathcal{D}[0, T], d^{(0)}\right)$ to $\mathbb{S}_{[0, T]}^{(q)}$.

\subsection{Implied $\mu, \beta$, and $\sigma$ surfaces}

Following this framework, we use the data from section 2.4 and the market option prices 31 for the underlying SPY asset to compute implied $\mu, \beta$, and $\sigma$ surfaces. Let $C_{i}^{(\mathrm{SPY}, \text { Market) }}\left(S_{0}^{(\mathrm{SPY})}, K, T, t, r_{f}\right)$ denote the price of the $i^{\text {th }}$ market call option contract on day $t$ where: $S_{0}^{(\mathrm{SPY})}$ is the spot price of the underlying SPY ETF; $K$ is the strike price; $T$ is the terminal time; and $r_{f}$ is the risk-free rate 32 For brevity, we simply refer to $C_{i}^{(\text {SPY,Market) }}$.

Applying equations (18), (19) and (20), we construct the GJR pricing tree's theoretical call option price for day $t$, denoted as $C_{i}^{(\mathrm{SPY}, \mathrm{GJR})}\left(S_{0}^{(\mathrm{SPY})}, K, T, t, r_{f}, \rho\right)$ For brevity, we refer to this as $C_{i}^{(\mathrm{SPY}, \mathrm{GJR})}(\rho)$, where $\rho=\mu, \beta$ or $\sigma$ is the parameter indicating the surface to be computed. For example, to obtain the implied $\mu$ surface, we use the estimated values for $\bar{\sigma}$ and $\bar{\beta}$ from section 2.4 and designate $\rho=\rho^{(\mu)}=\left(\mu \mid \bar{\sigma}^{(\alpha)}=\right.$ $\left.0.151, \bar{\beta}^{(\alpha)}=-0.978\right)$. We estimate the value for $\rho_{i}^{(\mu)}$ for the $i^{\text {th }}$ call option contract by

$$
\hat{\rho}_{i}^{(\mu)}=\arg \min \left\{\left(\frac{C_{i}^{(\mathrm{SPY}, \mathrm{GJR})}(\rho)-C_{i}^{(\mathrm{SPY}, \text { Market })}}{C_{i}^{(\mathrm{SPY}, \text { Market })}}\right)^{2}\right\}, i=1, \ldots, M .
$$

We proceed analogously using parameters $\rho^{(\beta)}$ and $\rho^{(\sigma)}$ to compute the $\beta$ and $\sigma$ surfaces.

Fig. [6] shows the results for the implied $\mu$ and $\beta$ surfaces, plotted in terms of moneyness $K / S$ and time to maturity $T$ (in days), where $K$ is the strike price and $S$ is the current spot price of the underlying asset. Of the two, the $\mu$ surface has the more complex behavior with different time-development of the surface as $K / S$ ranges from "in the money" to "out of the money" values. In contrast, values of $\hat{\beta}$ decrease on both sides of a ridge of values that generally aligns with $K / S \sim 1.1$. Values of $\beta$, ranging from -0.978 to 0.147 , are mostly negative indicating a negatively skewed pricing tree. Values of $\beta$ increase with time at any constant value of $K / S$.

\footnotetext{
${ }^{31}$ The SPY call option data was collected from Bloomberg Professional Services on 6/1/2021, 19:32 EST. The data set includes call options, with all strike values, having expiration date no later than $12 / 31 / 2021$. In total the data involves $M=1,913$ contracts with valid bid and ask quotes. The SPY spot price for this date and time was $\$ 419.67$.

${ }^{32}$ We use 10-year Treasury yield curve rates for risk-free rate values; the annual rate was $r_{f}=1.62 \%$ on $6 / 1 / 2021$.
} 


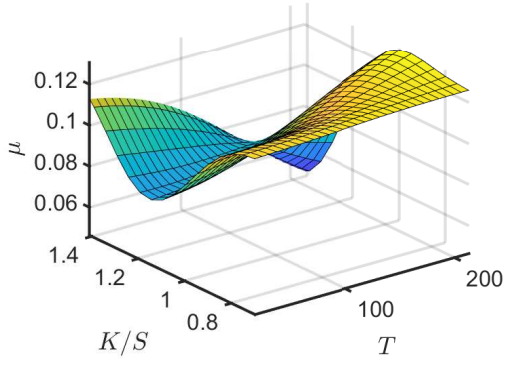

(a)

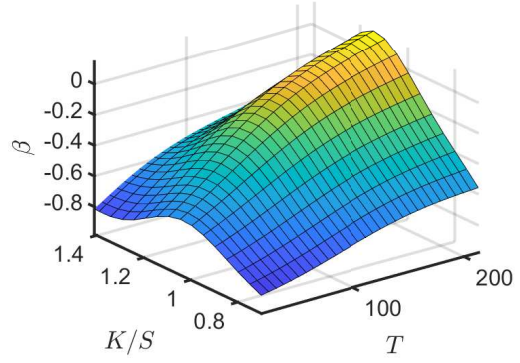

(b)

Figure 6: The implied (a) $\mu$ and (b) $\beta$ surfaces generated by the GJR pricing tree plotted as a function of moneyness $K / S$ and time to maturity $T$ (in days).

To evaluate the implied $\sigma$ surface, we also calculated the Black-Scholes implied volatility surface 33 $\sigma^{(\mathrm{BLS})}$ for the same option prices, and considered their difference using the percent deviation, Dev ${ }^{(\alpha, \mathrm{BLS})}=$ $100\left(\sigma-\sigma^{(\mathrm{BLS})}\right) / \sigma^{(\mathrm{BLS})}$. These surfaces are shown in Fig. 7. Both surfaces, $\sigma$ and $\sigma^{(\mathrm{BLS})}$, show similar volatility smiles and roughly similar values when $K / S>1$. However, the $\sigma$ surface increases more rapidly than $\sigma^{(\mathrm{BLS})}$ as $K / S$ moves deeper into the money.

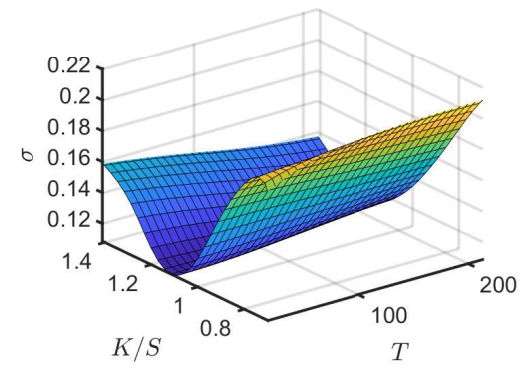

(a)

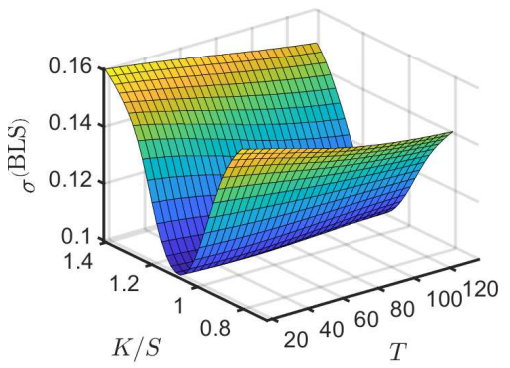

(b)

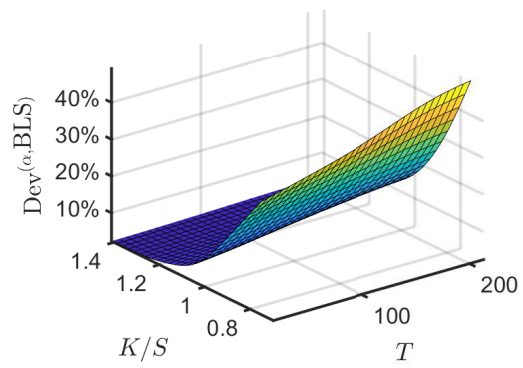

(c)

Figure 7: (a) The implied $\sigma$ surface generated by the GJR pricing tree. (b) The implied volatility surface, $\sigma^{(\mathrm{BLS})}$, using the Black-Scholes formula. (c) The deviation surface Dev ${ }^{(\alpha, B L S)}$. Each surface is plotted as a function of moneyness $K / S$ and time to maturity $T$ (in days).

\section{GJR option price with hedging transaction costs}

There is a vast literature on option pricing incorporating transaction costs 34 Discrete-time option pricing models have been studied under various assumptions regarding the types of the transaction costs incurred in trading the replicating self-financing portfolios 35 Here, we extend the GJR option pricing model to the case when the hedging is subject to transaction costs. Our approach in extending the GJR pricing tree model to include transaction costs is based on Merton's binomial option pricing model with transaction costs 36

\footnotetext{
${ }^{33}$ See Hull (2012, Chapter 15).

${ }^{34}$ Some basic references are: Leland (1985); Hodges and Neuberger $(1989)$; Bovle and Vorst (1992); Davis et al. (1993); Edirisinghe et al. (1993); Kabanov and Safarian (1997); Broadie et al. (1998); Kabanov and Stricker (2001); Palmer (2001); Lai and Lim (2009); and Guasoni et al. (2012)

${ }^{35}$ See Merton (1990, Chapter 14), Stettner (1997), Palmer (2001), Delbaen et al. (2002), Melnikov and Petrachenko (2005), and Chen et al. (2008).

${ }^{36}$ See Merton (1990, Chapter 14).
} 
Consider a market with three securities $(\mathcal{S}, \mathcal{B}, \mathcal{C})$ consisting of: (a) a stock $\mathcal{S}$ with pricing tree $\left\{S_{k \Delta t}^{(n)}, k=\right.$ $1, \ldots, n, n \Delta t=T\}$ given by (10), (b) a bond $\mathcal{B}$ with price dynamics given by (16), and (c) an ECC $\mathcal{C}$ with price dynamics given by (17). In contrast to the previous section, we assume that the hedger (the ECC-contract seller) trades the replicating risk-neutral portfolio $P_{k \Delta t}^{(n)}=D_{k \Delta t}^{(n)} S_{k \Delta t}^{(n)}-f_{k \Delta t}^{(n)}$ at a cost. Namely, given $\mathcal{F}_{k \Delta t}=\sigma\left(M_{1}^{\left(\alpha_{\Delta t}\right)}, \ldots, M_{k}^{\left(\alpha_{\Delta t}\right)}\right)$, the hedged portfolio at $(k+1) \Delta t$ is determined by

$$
\begin{aligned}
& P_{(k+1) \Delta t}^{(n, u, \lambda)}=D_{k \Delta t}^{(n)} S_{(k+1) \Delta t}^{(n, u)}+\lambda_{\Delta t} D_{k \Delta t}^{(n)}\left(S_{(k+1) \Delta t}^{(n, u)}-S_{k \Delta t}^{(n)}\right)-f_{(k+1) \Delta t}^{(n, u, \lambda)}, \\
& P_{(k+1) \Delta t}^{(n, d, \lambda)}=D_{k \Delta t}^{(n)} S_{(k+1) \Delta t}^{(n, d)}+\lambda_{\Delta t} D_{k \Delta t}^{(n)}\left(S_{(k+1) \Delta t}^{(n, d)}-S_{k \Delta t}^{(n)}\right)-f_{(k+1) \Delta t}^{(n, d, \lambda)} .
\end{aligned}
$$

In (22), $\lambda_{\Delta t}=\lambda^{(0)}+\lambda^{(1)} \sqrt{\Delta t}, \lambda^{(0)}>0, \lambda^{(1)} \in R$, is the hedging transaction cost (HTC). From (22), to leading order in $\Delta t$, it follows that

$$
D_{k \Delta t}^{(n)}=\frac{f_{(k+1) \Delta t}^{(n, u, \lambda)}-f_{(k+1) \Delta t}^{(n, d, \lambda)}}{2 S_{k \Delta t}^{(n)}\left(1+\lambda_{\Delta t}\right) \sigma \sqrt{\Delta t}} .
$$

As in (18) and (19), we obtain the risk-neutral valuation of the ECC,

$$
f_{k \Delta t}^{(n, \lambda)}=D_{k \Delta t}^{(n)} S_{k \Delta t}^{(n)}-e^{-r_{f} \Delta t} P_{(k+1) \Delta t}^{(n, u, \lambda)}=e^{-r_{f} \Delta t}\left(q_{n, k+1}^{(\lambda)} f_{(k+1) \Delta t}^{(n, u, \lambda)}+\left(1-q_{n, k+1}^{(\lambda)}\right) f_{(k+1) \Delta t}^{(n, d, \lambda)}\right),
$$

where the risk-neutral probability $q_{n, k+1}^{(\lambda)}$ has the form

$$
q_{n, k+1}^{(\lambda)}=\frac{1}{2}\left(1-\theta^{\left(\lambda^{(0)}\right)} \sqrt{\Delta t}-\beta(\sqrt{1+k}-\sqrt{k}) \sqrt{\frac{2 \Delta t}{\pi}}\right)-\frac{\lambda^{(1)} r_{f} \Delta t}{2 \sigma\left(1+\lambda^{(0)}\right)^{2}},
$$

with $\theta^{\left(\lambda^{(0)}\right)}=\left(\mu+\sigma^{2} / 2-r_{f} /\left(1+\lambda^{(0)}\right)\right) / \sigma 37$ In the special case, $\lambda^{(0)}=\lambda^{(1)}=0$, (25) coincides with (19).

Now consider the risk-neutral dynamics of the stock $\mathrm{S}$ in the presence of HTC. Conditional on $\mathcal{F}_{k \Delta t}, k=$ $1, \ldots, n-1$, the risk-neutral value of the stock price at $(k+1) \Delta t$ is determined by

$$
S_{(k+1) \Delta t}^{(n ; q, \lambda)}=\left\{\begin{array}{l}
S_{(k+1) \Delta t}^{(n, u ; q, \lambda)}=S_{k \Delta t}^{(n)} \exp (\mu \Delta t+\sigma \beta(\sqrt{k+1}-\sqrt{k}) \sqrt{2 / \pi} \Delta t+\sigma \sqrt{\Delta t}), \text { w.p. } q_{n, k+1}^{(\lambda)}, \\
S_{(k+1) \Delta t}^{(n, d ; q, \lambda)}=S_{k \Delta t}^{(n)} \exp (\mu \Delta t+\sigma \beta(\sqrt{k+1}-\sqrt{k}) \sqrt{2 / \pi} \Delta t-\sigma \sqrt{\Delta t}), \text { w.p. } 1-q_{n, k+1}^{(\lambda)},
\end{array}\right.
$$

where $q_{n, k+1}^{(\lambda)}$ is given by (25). Conditionally on $\mathcal{F}_{k \Delta t}$, the discrete risk-neutral log-return $R_{(k+1) \Delta t}^{(n ; q, \lambda)}=$ $\ln \left(S_{(k+1) \Delta t}^{(n ; q, \lambda)} / S_{k \Delta t}^{(n ; q, \lambda)}\right)$ has mean and variance

$$
\begin{aligned}
\mathbb{E}\left(R_{(k+1) \Delta t}^{(n ; q, \lambda)} \mid \mathcal{F}_{k \Delta t}\right) & =\left(\frac{r_{f}}{1+\lambda^{(0)}}-\frac{\sigma^{2}}{2}\right) \Delta t, \\
\operatorname{Var}\left(R_{(k+1) \Delta t}^{(n ; q, \lambda)} \mid \mathcal{F}_{k \Delta t}\right) & =\sigma^{2} \Delta t .
\end{aligned}
$$

Setting

$$
\mathbb{S}_{[0, T]}^{(n ; q, \lambda)}=\left\{S_{t}^{(n ; q, \lambda)}=S_{k \Delta t}^{(n ; q, \lambda)}, t \in[k \Delta t,(k+1) \Delta t), k=0, \ldots, n-1, S_{T}^{(n ; q)}=S_{n \Delta t}^{(n)}\right\}
$$

and

$$
\mathbb{S}_{[0, T]}^{(q, \lambda)}=\left\{S_{0} \exp \left(\left(\frac{r_{f}}{1+\lambda^{(0)}}-\frac{\sigma^{2}}{2}\right) t+\sigma B_{t}^{(q)}\right), t \in[0, T]\right\}
$$

where $\mathbb{B}_{[0, T]}^{(q)}=\left\{B_{t}^{(q)}, t \in[0, T]\right\}$ is a standard BM, we have that $\mathbb{S}_{[0, T]}^{(n, q)}$ converges weakly in $\left(\mathcal{D}[0, T], d^{(0)}\right)$ to $\mathbb{S}_{[0, T]}^{(q)}$. From (28), it follows that the value of $\lambda^{(1)}$ in the HTC is irrelevant in continuous-time trading.

\footnotetext{
${ }^{37}$ Formula (23) for the delta-position $D_{k \Delta t}^{(n)}$ is similar to formula (14.2a) in Merton (1990, Chapter 14).
} 
However, for every fixed $\Delta t$, the risk-neutral tree (20) will exhibit the behavior of a geometric SBM and the value of $\lambda^{(1)}$ becomes relevant, as evident from (24).

Remark: It appears natural to assume that HTC could be asymmetric; that is, instead of (22), we could assume that

$$
\begin{aligned}
& P_{(k+1) \Delta t}^{(n, u, \lambda)}=D_{k \Delta t}^{(n)} S_{(k+1) \Delta t}^{(n, u)}+\lambda_{\Delta t}^{(u)} D_{k \Delta t}^{(n)}\left(S_{(k+1) \Delta t}^{(n, u)}-S_{k \Delta t}^{(n)}\right)-f_{(k+1) \Delta t}^{(n, u, \lambda)}, \\
& P_{(k+1) \Delta t}^{(n, d, \lambda)}=D_{k \Delta t}^{(n)} S_{(k+1) \Delta t}^{(n, d)}+\lambda_{\Delta t}^{(d)} D_{k \Delta t}^{(n)}\left(S_{(k+1) \Delta t}^{(n, d)}-S_{k \Delta t}^{(n)}\right)-f_{(k+1) \Delta t}^{(n, d, \lambda)},
\end{aligned}
$$

with $\lambda_{\Delta t}^{(u)} \neq \lambda_{\Delta t}^{(d)}$, where

$$
\begin{aligned}
& \lambda_{\Delta t}^{(u)}=\lambda^{(0, u)}+\lambda^{(1, u)} \sqrt{\Delta t}, \lambda^{(0, u)}>0, \lambda^{(1, u)} \in R, \\
& \lambda_{\Delta t}^{(d)}=\lambda^{(0, d)}+\lambda^{(1, d)} \sqrt{\Delta t}, \lambda^{(0, d)}>0, \lambda^{(1, d)} \in R .
\end{aligned}
$$

A close inspection shows that, as $\Delta t \downarrow 0$, (29) leads to a pricing model which is not arbitrage free. However, for fixed $\Delta t$, the model (29) is arbitrage free and an expression for the risk-neutral probability $q_{n, k+1}^{(\lambda)}$ similar to formula (24) can be readily obtained 38

\subsection{Implied surfaces with the inclusion of HTC}

As in section 3.1, we numerically illustrate the HTC and its limiting behavior using the same data set, including closing and call option prices, for the underlying asset SPY. Following the notation in section 3.1 we set the parameter $\rho=\rho^{(\lambda)}=\left(\lambda^{(0)}, \lambda^{(1)} \mid \bar{\mu}^{(\alpha)}, \bar{\beta}^{(\alpha)}, \bar{\sigma}^{(\alpha)}\right)$. From (24), (25) and (26), we construct the GJR tree option price $C_{i}^{\text {(SPY,GJR) }}\left(\rho^{(\lambda)}\right)$. We find the optimal solution for $\rho^{(\lambda)}$ by minimizating the relative mean-square error (relMSE)

$$
\min _{\lambda^{(0)}>0, \lambda^{(1)} \in R} \operatorname{relMSE}=\min _{\lambda^{(0)}>0, \lambda^{(1)} \in R} \frac{1}{M} \sum_{i=1}^{M}\left(\frac{C_{i}^{(\mathrm{SPY}, \mathrm{GJR})}(\rho)-C_{i}^{(\mathrm{SPY}, \text { Market })}}{C_{i}^{\text {(SPY,Market })}}\right)^{2} .
$$

The resulting minimizing values are relMSE $=0.433$ for $\lambda^{(0)}=28.8$ and $\lambda^{(1)}=0.29739$ Using the parameters $\rho^{\left(\lambda^{(0)}\right)}=\left(\lambda^{(0)} \mid \bar{\mu}^{(\alpha)}, \bar{\beta}^{(\alpha)}, \bar{\sigma}^{(\alpha)}, \lambda^{(1)}=0.297\right)$ and $\rho^{\left(\lambda^{(1)}\right)}=\left(\lambda^{(1)} \mid \bar{\mu}^{(\alpha)}, \bar{\beta}^{(\alpha)}, \bar{\sigma}^{(\alpha)}, \lambda^{(0)}=28.8\right)$, we reran the minimization problem (21) and computed the $\lambda^{(0)}$ and $\lambda^{(1)}$ surfaces shown in Fig. 8 . Both surfaces have a similar ridge shape, but of vastly different scales. The value of $\lambda^{(1)}$ is constant through five computed digits, whereas $\lambda^{(0)}$ varies significantly in the first computed digit. Thus, for these values of $\bar{\mu}^{(\alpha)}, \bar{\beta}^{(\alpha)}$ and $\bar{\sigma}^{(\alpha)}$, the constant value model, $\lambda_{\Delta t}=\lambda^{(0)}$, would suffice in (22).

To ascertain the effects of adding HTC, we computed the implied $\mu, \beta$, and $\sigma$ surfaces using the minimizing values for $\lambda^{(0)}$ and $\lambda^{(1)}$. (For example, for the $\mu$ surface we estimated values for $\rho^{(\mu)}=\left(\mu \mid \bar{\sigma}^{(\alpha)}=\right.$ $\left.0.151, \bar{\beta}^{(\alpha)}=-0.978, \lambda^{(0)}=28.8, \lambda^{(1)}=0.297\right)$ using (21).) To compare the results with those reported in section 3.1, we also computed the percent deviation surfaces, $\rho^{(\mathrm{dev})}=100\left(\rho^{(\mathrm{HTC})}-\rho^{(\mathrm{GJR})}\right) / \rho^{(\mathrm{GJR})}$ for $\rho=\{\mu, \sigma\}$, where $\rho^{(\mathrm{GJR})}$ refers to the surface computed for the GJR model without HTC in section 3.1 and $\rho^{\text {(HTC) }}$ refers to the surface computed for the GJR model with HTC. To avoid division by zero, for the $\beta$ surface comparison we plot the difference surface $\beta^{(\mathrm{diff})}=\beta^{(\mathrm{HTC})}-\beta^{\text {(GJR) }}$. The results are shown in Fig. 9 , Inclusion of HTC has the effect of differentially lowering $\mu$ values (as expected since the trader is expending money on fees), most noticeably along the coordinate $K / S \sim 1.2$. Similarly expected, inclusion of HTC differentially raises the volatility surface. HTC differentially raises $\beta$ values, with greater increases arising as $K / S$ values move more "into the money", resulting in larger regions of the phase space where the pricing tree is positively skewed.

\footnotetext{
${ }^{38}$ Discrete-time market models, which are free of arbitrages, but for which the corresponding continuous-time market model is not arbitrage-free, are known; see, for example, Karandikar and Rachev (1995) and Hurst et al. (1999). In those models, the discrete equivalent martingale measure explodes in the limit. Similarly, if we assume that (29) holds, the limiting martingale measure does not exist.

${ }^{39}$ The three terms on the right-hand side of $(22)$ have the form $D S+\lambda D \Delta S-f$, where the transaction cost term is $\lambda D \Delta S$. The estimated values of $\lambda^{(0)}$ and $\lambda^{(1)}$ yield $\lambda \approx \lambda^{(0)}=28.8$. If daily stock price changes for the SPY fund are around $0.1 \%$ of the fund price (i.e. $\Delta S \sim 10^{-3} S$ ), the transaction cost term would have the value $0.0288 D S$, indicating transactions costs contribute $\sim 3 \%$ to the total price of the hedged portfolio.
} 

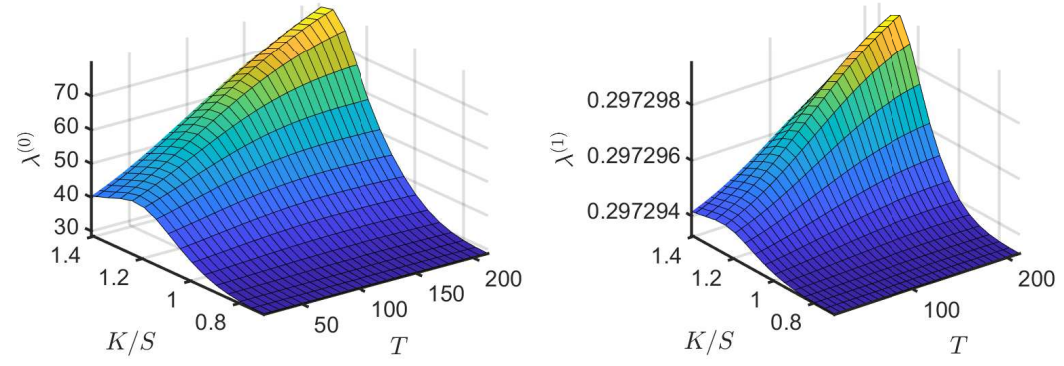

Figure 8: The implied $\lambda^{(0)}$ and $\lambda^{(1)}$ surfaces based on the GJR pricing tree with HTC plotted as a function of moneyness $K / S$ and time to maturity $T$ (in days).

\section{$5 \quad$ Fitting the GJR pricing tree to a market driver}

In this section we explore possibilities for fitting the pricing tree model, $S_{k \Delta t}^{(n)}, k=1, \ldots, n$, to a market driver that affects the price dynamics, $S_{k \Delta t}^{(n, \mathcal{S})}$, of stock $\mathcal{S}$. We consider both endogenous and exogenous approaches. In the endogenous approach, the discrete dynamics for the price of $\mathcal{S}$ is modeled by

$$
\begin{aligned}
S_{k \Delta t}^{(n, \text { endo })} & =S_{0} \exp \left(v_{k}^{(0, \mathcal{S})} \Delta t+M_{k}^{(\text {endo })} \sigma^{(0, \mathcal{S})} \sqrt{\Delta t}\right), \\
v_{k}^{(0, \mathcal{S})} & =k \mu^{(0, \mathcal{S})}+\sigma^{(0, \mathcal{S})} \beta^{(0, \mathcal{S})}(\sqrt{2 k / \pi}-1), k=1, \ldots, n,
\end{aligned}
$$

with the path $M_{1}^{(\text {endo })}, \ldots, M_{k}^{(\text {endo) }}$ of the Markov chain $\mathbb{M}^{(\text {endo) }}$ determined by 40

$$
M_{k}^{(\text {endo })}=\sum_{j=1}^{k} \operatorname{sign}\left(r_{j \Delta t}^{(n, \mathcal{S})}\right), j=1, \ldots, k ; M_{0}^{(\text {endo })}=0,
$$

where $r_{j \Delta t}^{(n, \mathcal{S})}=\ln \left(S_{j \Delta t}^{(n, \mathcal{S})} / S_{(j-1) \Delta t}^{(n, \mathcal{S})}\right)$ is the market's daily log-return for stock $\mathcal{S}$. In (31), the parameters $\sigma^{(0, \mathcal{S})}, \mu^{(0, \mathcal{S})}$ and $\beta^{(0, \mathcal{S})}$ are determined for the initial time $k \Delta t=0$ from equations (14) and (15) using the cumulative return data for $\mathcal{S}$ applicable at the chosen start date $k \Delta t=0$. The values obtained from (14) and (15) are also smoothed using the moving-window averaging procedure introduced in section 2.4. This will be demonstrated explicitly by numerical example in section 5.1 .

The endogenous approach is limited while the exogenous approach assumes that the $M_{k}^{\left(\alpha_{\Delta t}\right)}$ are determined by the upward and downward movements of a more general market driver. For concreteness, we initially assume the market driver is a factor model for the price dynamics of $\mathcal{S}$; in particular we consider the Fama-French five-factor asset pricing model.

Fama-French five-factor model W1 With $r_{j \Delta t}^{(n, \mathcal{S})}$ being the market log-return of stock $\mathcal{S}$ for the period $[j \Delta t,(j+1) \Delta t)$, the coefficients of the terms in the Fama-French factor return,

$$
r_{j \Delta t}^{(n, F)}=r_{f, j \Delta t}+\mathfrak{a}+\mathbb{b}\left(r_{j \Delta t}^{(n, M)}-r_{f, j \Delta t}\right)+\$ r_{j \Delta t}^{(n, S M B)}+\mathbb{h} r_{j \Delta t}^{(n, H M L)}+\mathbb{r} r_{j \Delta t}^{(n, R M W)}+\mathbb{C} r_{j \Delta t}^{(n, C M A)},
$$

are determined from the regression

$$
r_{j \Delta t}^{(n, \mathcal{S})}=r_{j \Delta t}^{(n, F)}+\epsilon_{j \Delta t}, \quad j=0,1, \ldots, m-1 .
$$

In (32),$r_{f, j \Delta t}$ is the risk-free rate in period $[j \Delta t,(j+1) \Delta t)$ and the remaining Fama-French factors are:

\footnotetext{
${ }^{40}$ Here $\operatorname{sign}(x)=\left\{\begin{array}{l}1, \text { if } x \geq 0 \\ -1, \text { if } x<0\end{array}\right.$.

${ }^{41}$ See Fama and French $(2012,2015,2017)$.
} 


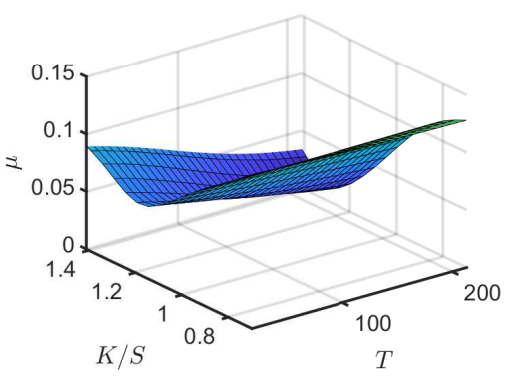

(a)

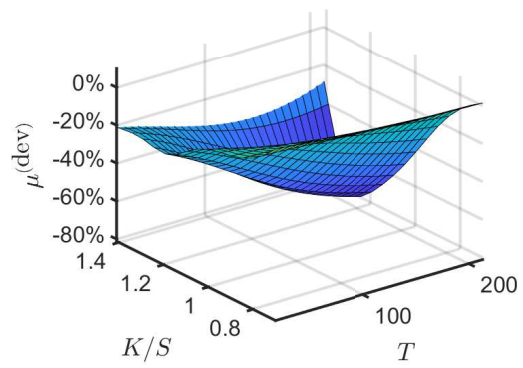

(d)

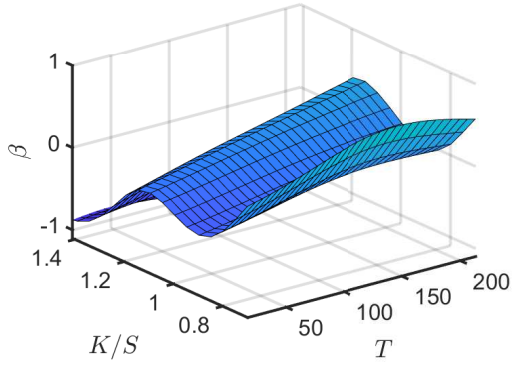

(b)

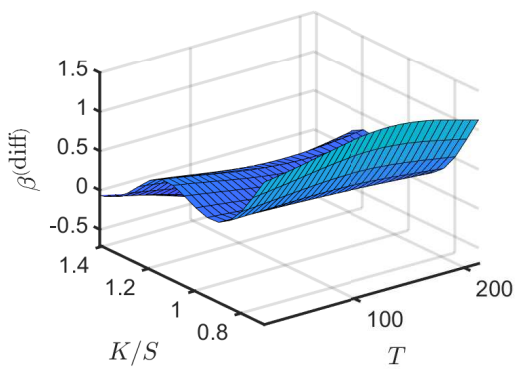

(e)

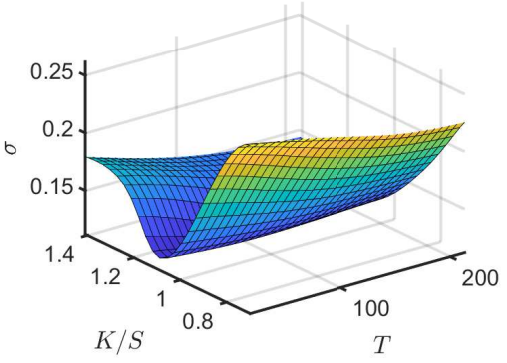

(c)

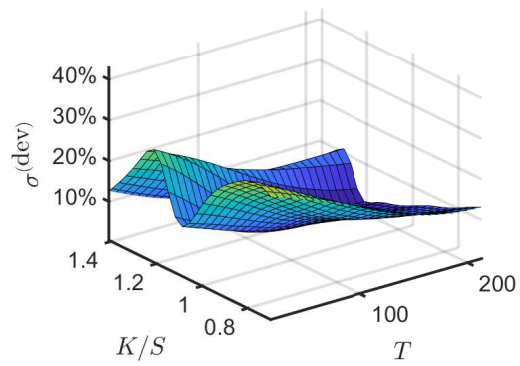

(f)

Figure 9: The implied (a) $\mu$, (b) $\beta$ and (c) $\sigma$ surfaces generated by the GJR pricing tree with HTC added. The percent deviation surfaces $\mu^{(\mathrm{dev})}$ and $\sigma^{(\mathrm{dev})}$ are shown in (d) and (f) respectively, while (e) displays the difference surface $\beta^{\text {(diff) }}$. Each surface is plotted as a function of moneyness $K / S$ and time to maturity $T$ (in days).

$\mathbb{F}_{1}: r_{j \Delta t}^{(n, M)}$ (representing the market-factor effect) is the return over the period $[j \Delta t,(j+1) \Delta t)$ of the capitalization weighted stock market to which $\mathcal{S}$ belongs;

$\mathbb{F}_{2}: r_{j \Delta t}^{(n, S M B)}$ (representing the size-factor effect) is the difference in the return of a portfolio in $\mathcal{M}$ specializing in (a) small-cap and (b) large-cap stocks;

$\mathbb{F}_{3}: r_{j \Delta t}^{(n, H M L)}$ (representing the value-factor effect) is the difference of the returns of a portfolio in $\mathcal{M}$ with (a) high and (b) small book-to-market ratios;

$\mathbb{F}_{4}: r_{j \Delta t}^{(n, R M W)}$ (representing the profitability-premium-factor effect) is the difference of the return of a portfolio in $\mathcal{M}$ with (a) high and (b) low profitability; and

$\mathbb{F}_{5}: r_{j \Delta t}^{(n, C M A)}$ (representing the investment-attitude-factor effect) is the difference of the return of a portfolio of stocks in $\mathcal{M}$ issued by firms that invest (a) conservatively and (b) aggressively.

Let $j \Delta t, j=1, \ldots, m$ denote a time period $\tau_{1}$ and $j=m+1, \ldots, m+n$ denote the following time period $\tau_{2}$. Let $\hat{r}_{j \Delta t}^{(n, F)}, j=1, \ldots, m$, denote sample return values (factor returns) for (32) obtained from the regression (33) over the period $\tau_{1}$, and $\hat{R}_{j \Delta t}^{(n, F)}$ denote the resultant cumulative factor returns. Using the cumulative factor returns on the left-hand side of (14) and (15), and the moving-window averaging procedure introduced in section 2.4, we obtain estimates $\bar{\sigma}^{(F)}, \bar{\mu}^{(F)}$ and $\bar{\beta}^{(F)}$ for the values of the parameters $\sigma, \mu$ and $\beta$ at timestep $m \Delta t$. Let $k \Delta t, k=1, \ldots, n$, where $k=j-m$, label the trading days in time period $\tau_{2}$. Then $\bar{\sigma}^{(F)}, \bar{\mu}^{(F)}$ and $\bar{\beta}^{(F)}$ correspond to parameter values for $k \Delta t=0$. 
We model the discrete dynamics for the factor-driven price over the period $\tau_{2}$ by

$$
\begin{aligned}
S_{k \Delta t}^{(n, \text { exo })} & =S_{0} \exp \left(v_{k}^{(F)} \Delta t+M_{k}^{\left(\bar{\alpha}^{(F)}, \operatorname{exo}\right)} \bar{\sigma}^{(F)} \sqrt{\Delta t}\right), \\
v_{k}^{(F)} & =k \bar{\mu}^{(F)}+\bar{\sigma}^{(F)} \bar{\beta}^{(F)}(\sqrt{2 k / \pi}-1),
\end{aligned}
$$

where $\bar{\alpha}_{\Delta t}^{(F)}=\left(1+\bar{\beta}^{(F)} \sqrt{\Delta t}\right) / 2$. To estimate the path $M_{k}^{\left(\bar{\alpha}^{(F)} \text {,exo }\right)}$, we generate a large ensemble of sample paths $\left\{M_{k}^{\left(\bar{\alpha}^{(F)}\right)}, k=1, \ldots, n\right\} \cdot M_{k}^{\left(\bar{\alpha}^{(F)}, \text { exo }\right)}$ is then chosen as that path that minimizes the relMSE between the two sides of (34) when the stock prices $S_{k \Delta t}^{(n, \mathcal{S})}$ are used on the left-hand side of (34). This exogenous method is also demonstrated in section 5.1

\subsection{Numerical example of the endogenous and exogenous approaches}

We demonstrate the endogenous and exogenous approaches using the daily return series for the stock $\mathcal{S}=$ MSFT. The price data set42 covers the period 4/30/2015 tthrough 4/30/2021. Since the estimation (equations (14) and (15)) and averaging procedures of section 2.4 for $\sigma, \mu$ and $\beta$ require one-year moving windows, we divide the data set into two time periods, $\tau_{1}=4 / 31 / 2015$ through $4 / 28 / 2017$ and $\tau_{2}=5 / 1 / 2017$ through $4 / 30 / 2021$. MSFT data on the close of $4 / 28 / 2017$ in $\tau_{1}$ serves as $t=0$ data for $\tau_{2}$; the closing price of MSFT on $4 / 28 / 2017$ serves as $S_{0}$ for the period $\tau_{2}$ and cumulative returns over $\tau_{2}$ are then computed relative to this $S_{0}$. And applying the estimation and averaging procedures of section 2.4 to the cumulative log-return data for MSFT over $\tau_{1}$, we obtain estimates $\bar{\sigma}^{(0, \mathrm{MSFT})}, \bar{\mu}^{(0, \mathrm{MSFT})}$ and $\bar{\beta}^{(0, \mathrm{MSFT})}$ determined for the close of trading on $4 / 28 / 2017$. These values, along with $\bar{\alpha}^{(0, \text { MSFT })}$ and the value of the relMSE, are given in Table 11 Using $\bar{\sigma}^{(0, \mathrm{MSFT})}, \bar{\mu}^{(0, \mathrm{MSFT})}$ and $\bar{\beta}^{(0, \mathrm{MSFT})}$ in (31) we obtain an endogenous estimate $S_{k \Delta t}^{(n, \text { endo) }}$ for the cumulative price dynamics of MSFT over the period $\tau_{2}$.

\begin{tabular}{cccccc}
\hline \multirow{2}{*}{ endogenous } & $\bar{\mu}^{(0, \mathrm{MSFT})}$ & $\bar{\sigma}^{(0, \mathrm{MSFT})}$ & $\bar{\beta}^{(0, \mathrm{MSFT})}$ & $\bar{\alpha}^{(0, \mathrm{MSFT})}$ & relMSE \\
& $7.38 \cdot 10^{-4}$ & $8.91 \cdot 10^{-2}$ & -4.08 & 0.371 & $1.78 \cdot 10^{-3}$ \\
\hline \multirow{2}{*}{ exogenous } & $\bar{\mu}^{(F)}$ & $\bar{\sigma}^{(F)}$ & $\bar{\beta}^{(F)}$ & $\bar{\alpha}^{(F)}$ & $\mathrm{relMSE}$ \\
& $2.31 \cdot 10^{-4}$ & $7.01 \cdot 10^{-3}$ & -11.2 & 0.147 & $3.22 \cdot 10^{-3}$ \\
\hline
\end{tabular}

Table 1: Parameters estimates from the endogenous and exogenous estimation procedures

For the exogenous approach, we use the Fama-French five-factor model outlined in the previous section as the market driver. The Fama-French factor returns, $r_{k \Delta t}^{(n, F)}$ in (32), were computed over the period $\tau_{1}$ using a robust form for the regression (33) 43 The values of the coefficients, as well as the R-square and root mean square-error (RMSE) values, are given in Table 2. The sample return $\hat{r}_{k \Delta t}^{(n, F)}$ and price $\hat{S}_{k \Delta t}^{(n, F)}$ series computed from these coefficients are presented in Fig. 10 where they are compared with the market return $r_{k \Delta t}^{(n, \mathrm{MSFT})}$ and price $S_{k \Delta t}^{(n, \mathrm{MSFT})}$ series over the period $\tau_{1}$. The significant differences between the series $\hat{S}_{k \Delta t}^{(n, F)}$ and $S_{k \Delta t}^{(n, \mathrm{MSFT})}$ are indicative of issues with applying market driver analyses to stock returns. Since the Fama-French model is based upon a regression fit to return values, errors produced in return values have large-time decay structure and lead ultimately to significant cumulative differences in price performance.

The parameter estimates $\bar{\mu}^{(F)}, \bar{\sigma}^{(F)}, \bar{\beta}^{(F)}$, and $\bar{\alpha}^{(F)}$ obtained for 4/28/2017 using the cumulative FamaFrench returns over $\tau_{1}$ are given in Table 1. These values generally differ by a factor of $\sim 3$ compared to the corresponding endogenous variables, with the exception of a factor of $\sim 13$ difference between $\bar{\sigma}^{(F)}$ and $\bar{\sigma}^{(0, \mathrm{MSFT})}$. Using the estimated value $\bar{\alpha}^{(F)}$, we generate $10^{6}$ scenarios of the sample path $M_{k}^{\left(\bar{\alpha}^{(F)}\right)}$ covering

\footnotetext{
${ }^{42}$ MSFT market closing prices from Bloomberg Professional Services. Return values for the Fama-French factors from the U.S. Research Returns Data Center (https://mba.tuck.dartmouth.edu/pages/faculty/ken.french/Data_Library.html).

${ }^{43}$ Motivated by results in Knez and Readv (1997), we choose robust regression in estimating the parameters of the FamaFrench five-factor model.
} 


\begin{tabular}{|c|c|c|c|c|c|c|c|}
\hline $\mathrm{a}$ & $\mathrm{b}$ & $\Phi$ & hh & r & $\mathbb{C}$ & R-square & RMSE \\
\hline$-6.5 \cdot 10^{-4}$ & $1.2 \cdot 10^{-2}$ & $-3.9 \cdot 10^{-3}$ & $-4.0 \cdot 10^{-4}$ & $3.0 \cdot 10^{-3}$ & $-5.8 \cdot 10^{-3}$ & 0.58 & $9.1 \cdot 10^{-3}$ \\
\hline
\end{tabular}

Table 2: Parameter estimates from the regression (33)
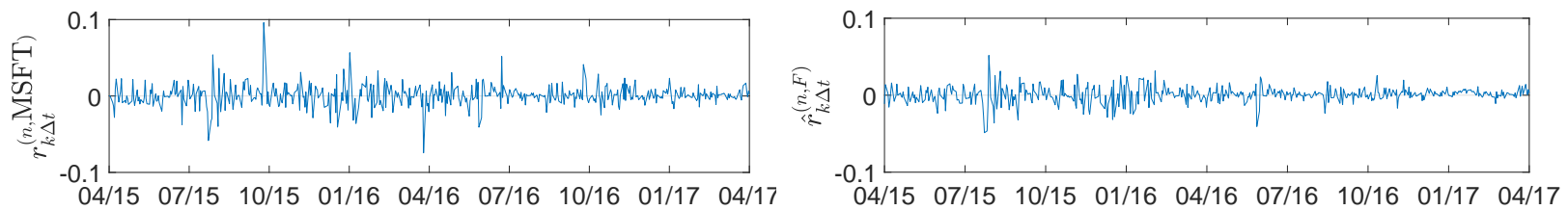

(a)

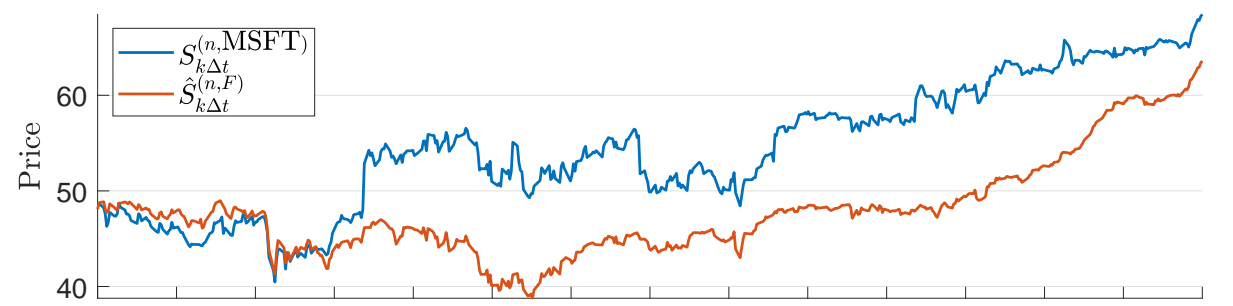

04/15 06/15 08/15 10/15 11/15 01/16 03/16 04/16 06/16 08/16 10/16 11/16 01/17 03/17

(b)

Figure 10: (a) Comparison of the MSFT market (a) return series $r_{k \Delta t}^{(n, \mathrm{MSFT})}$ and (b) price series $S_{k \Delta t}^{(n, \mathrm{MSFT})}$ with with the return series $\hat{r}_{k \Delta t}^{(n, F)}$ and corresponding price series $\hat{S}_{k \Delta t}^{(n, F)}$ produced by a regression fit to the Fama-French five-factor model over the period $\tau_{1}$.

the time period $\tau_{2}$. Using the price data $S_{k \Delta t}^{(n, \mathrm{MSFT})}$ for $\tau_{2}$ on the left-hand side of (34), the optimal path $M_{k}^{\left(\bar{\alpha}^{(F)} \text {,exo }\right)}$ was selected as the path scenario that minimized the relMSE between the left- and right-hand sides. The exogenous price series estimate $S_{k \Delta t}^{(n, \text { exo })}$ for $\tau_{2}$ is then computed from (34) using $M_{k}^{\left(\bar{\alpha}^{(F)} \text {,exo) }\right.}$ on the right-hand side.

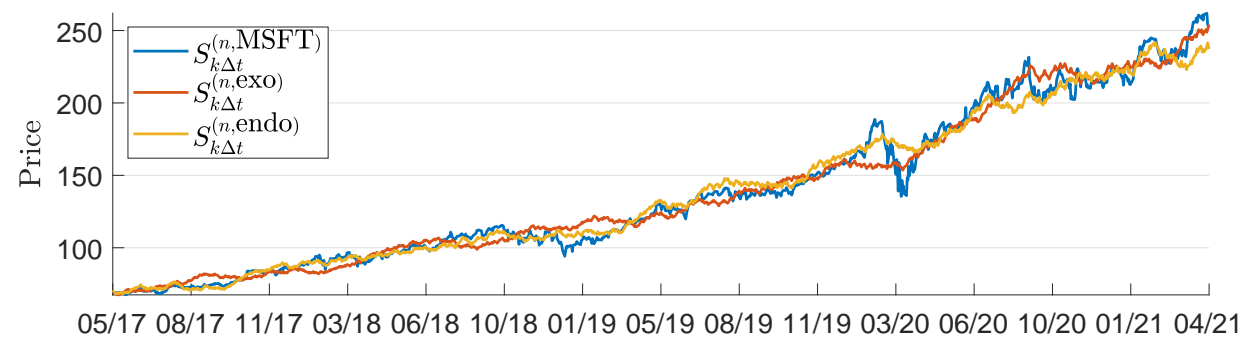

Figure 11: Comparisons of the MSFT market price series $S_{k \Delta t}^{(n, \mathrm{MSFT})}$ to the estimated endogenous, $S_{k \Delta t}^{(n, \text { endo) }}$, and exogenous, $S_{k \Delta t}^{(n, \text { exo) }}$, price dynamics over the time period $\tau_{2}$.

The cumulative price $S_{k \Delta t}^{(n, \mathrm{MSFT})}$ over $\tau_{2}$ is compared with the estimates $S_{k \Delta t}^{(n, \text { endo) }}$ and $S_{k \Delta t}^{(n, \text { exo })}$ in Fig. 11 . Both estimates produce reasonable agreement to the actual price data; however both significantly smooth and decrease the impact of the Covid-19 pandemic; the exogenous method showing less impact from the pandemic than the endogenous method. 


\subsection{Inclusion of the higher moment SBM process $\mathbb{C}_{t}^{(\alpha, n)}$}

For a general market driver, we extend to the full functionality of the CYSIP by adding the higher moment SBM process $\mathbb{C}_{t}^{(\alpha, n)}$ into our model 44 According to (34), $S_{k \Delta t}^{(n, \text { exo) }}$ is adapted to the filtration

$$
\mathbb{F}^{\left(n ; \mathbb{M}^{(\alpha \Delta t)}\right)}=\left\{\mathcal{F}_{k}^{\left(n ; \mathbb{M}^{(\alpha \Delta t)}\right)}=\sigma\left(M_{j}^{(\alpha \Delta t)} ; j=1, \ldots, k\right), k=1, \ldots, n, \mathcal{F}_{k}^{\left(n ; \mathbb{M}^{(\alpha \Delta t)}\right)}=\{\varnothing, \Omega\}\right\} .
$$

Paralleling the development for the lower moment SBM process in section 2.2, with $n \Delta t=T$ we set $M_{0}^{\left(\alpha_{\Delta t}, n\right)}=0, M_{k}^{\left(\alpha_{\Delta t}, n\right)}=n^{-1 / 2} M_{k}^{\left(\alpha_{\Delta t}\right)}$, and $X_{k / n}^{\left(\alpha_{\Delta t}, n\right)}=\sum_{i=1}^{k} M_{i}^{\left(\alpha_{\Delta t}, n\right)}, k=1, \ldots, n$. Let $B_{t}^{\left(\alpha_{\Delta t}, n\right)}, t \geq 0$ be a random process with piecewise linear trajectories having vertexes $\left(k / n, \mathbb{B}_{k / n}^{\left(\alpha_{\Delta t}, n\right)}\right), k=1, \ldots, n$, where $\mathbb{B}_{k / n}^{\left(\alpha_{\Delta t}, n\right)}=X_{k / n}^{\left(\alpha_{\Delta t}, n\right)}$. Let $h: R \rightarrow R$ be a piecewise continuous function and define $Y_{k / n}^{\left(\alpha_{\Delta t}, n\right)}=$ $\sum_{i=1}^{k} h\left(X_{(i-1) / n}^{\left(\alpha_{\Delta t}, n\right)}\right)\left(X_{i / n}^{\left(\alpha_{\Delta t}, n\right)}-X_{(i-1) / n}^{\left(\alpha_{\Delta t}, n\right)}\right), k \in \mathcal{N}, n \in \mathcal{N}$. Define $\mathbb{C}_{t}^{\left(\alpha_{\Delta t}, n ; h\right)}, t \geq 0$ to be a random process with the piecewise linear trajectories having vertexes $\left(k / n, \mathbb{C}_{k / n}^{\left(\alpha_{\Delta t}, n ; h\right)}\right), k \in \mathcal{N}, n \in \mathcal{N}$, where $\mathbb{C}_{k / n}^{\left(\alpha_{\Delta t}, n ; h\right)}=$ $Y_{k / n}^{\left(\alpha_{\Delta t}, n ; h\right)}$. Then, for a fixed, relatively small value of $\Delta t$, the bivariate process $\left(\mathbb{B}_{t}^{\left(\alpha_{\Delta t}, n\right)}, \mathbb{C}_{t}^{\left(\alpha_{\Delta t}, n ; h\right)}\right), t \geq 0$, approaches $\left(B_{t}^{\left(\alpha_{\Delta t}\right)}, C_{t}^{\left(\alpha_{\Delta t} ; h\right)}\right)$, where $B_{t}^{\left(\alpha_{\Delta t}\right)}$ is a SBM and $C_{t}^{\left(\alpha_{\Delta t} ; h\right)}=\int_{0}^{t} h\left(B_{s}^{\left(\alpha_{\Delta t}\right)}\right) d B_{s}^{\left(\alpha_{\Delta t}\right)}$. However, as $n \uparrow \infty$ and $\Delta t \downarrow 0,\left(\mathbb{B}_{t}^{\left(\alpha_{\Delta t}, n\right)}, \mathbb{C}_{t}^{\left(\alpha_{\Delta t}, n\right)}\right)$ converges in law to $\left(B_{t}, C_{t}\right)$ for $t \geq 0$, where $B_{t}$ is a BM and $C_{t}=\int_{0}^{t} h\left(B_{s}\right) d B_{s} 45$

Next, consider the following two processes in $\mathcal{D}[0, T]$ :

$$
\begin{aligned}
\mathbb{B}_{[0, T]}^{\left(\alpha_{\Delta t}, n\right)} & =\left\{\mathbb{B}_{t}^{\left(\alpha_{\Delta t}, n\right)}=\sqrt{T} X_{k / n}^{\left(\alpha_{\Delta t}, n\right)}, t \in[k \Delta t,(k+1) \Delta t), \mathbb{B}_{1}^{\left(\alpha_{\Delta t}, n\right)}=X_{1}^{\left(\alpha_{\Delta t}, n\right)}\right\}, \\
\mathbb{C}_{[0, T]}^{\left(\alpha_{\Delta t}, n, h\right)} & =\left\{\begin{array}{l}
\mathbb{C}_{t}^{\left(\alpha_{\Delta t}, n ; h\right)}=\sum_{i=1}^{k} h\left(\sqrt{T} X_{(i-1) / n}^{\left(\alpha_{\Delta t}, n\right)}\right)\left(\sqrt{T} X_{i / n}^{\left(\alpha_{\Delta t}, n\right)}-\sqrt{T} X_{(i-1) / n}^{\left(\alpha_{\Delta t}, n\right)}\right), t \in[k \Delta t,(k+1) \Delta t), \\
\mathbb{C}_{1}^{\left(\alpha_{\Delta t}, n\right)}=Y_{1}^{\left(\alpha_{\Delta t}, n\right)}
\end{array}\right\} .
\end{aligned}
$$

Then, for large $n$, the bivariate process $\left(\mathbb{B}_{[0, T]}^{\left(\alpha_{\Delta t}, n\right)}, \mathbb{C}_{[0, T]}^{\left(\alpha_{\Delta t}, n ; h\right)}\right)$ approaches $\left(\mathbb{B}_{[0, T]}^{\left(\alpha_{\Delta t}\right)}, \mathbb{C}_{[0, T]}^{\left(\alpha_{\Delta t} ; h\right)}\right)$ in $\mathcal{D}[0, T]$. $\mathcal{D}[0, T]$, where $\mathbb{B}_{[0, T]}^{\left(\alpha_{\Delta t}\right)}=\left\{B_{t}^{\left(\alpha_{\Delta t}\right)}, t \in[0, T]\right\}$ is a SBM on $[0, T]$ and $\mathbb{C}_{[0, T]}^{\left(\alpha_{\Delta t} ; h\right)}=\left\{C_{t}^{\left(\alpha_{\Delta t} ; h\right)}, t \in[0, T]\right\}$. Ultimately, as $n \uparrow \infty,\left(\mathbb{B}_{[0, T]}^{\left(\alpha_{\Delta t}, n\right)}, \mathbb{C}_{[0, T]}^{\left(\alpha_{\Delta t}, n ; h\right)}\right)$ converges weakly in $\mathcal{D}[0, T] \cdot \mathcal{D}[0, T]$ to $\left(\mathbb{B}_{[0, T]}, \mathbb{C}_{[0, T]}^{(h)}\right)$, where $\mathbb{B}_{[0, T]}=\left\{B_{t}, t \in[0, T]\right\}$ is a $\mathrm{BM}$ on $[0, T]$ and $\mathbb{C}_{[0, T]}^{(h)}=\left\{C_{t}^{(h)}=\int_{0}^{t} h\left(B_{s}\right) d B_{s}, t \in[0, T]\right\}$.

Now define the stock price discrete dynamics as a functional of $\mathbb{B}_{[0, T]}^{\left(\alpha_{\Delta t}, n\right)}$ and $\mathbb{C}_{[0, T]}^{\left(\alpha_{\Delta t}, n ; h\right)}$. Let

$$
\mathbb{S}_{[0, T]}^{\left(\alpha_{\Delta t}, n ; h\right)}=\left\{\begin{array}{l}
S_{t}^{\left(\alpha_{\Delta t}, n ; h\right)}=S_{0} \exp \left(v k \Delta t+\sigma \mathbb{B}_{t}^{\left(\alpha_{\Delta t}, n\right)}+\gamma \mathbb{C}_{t}^{\left(\alpha_{\Delta t}, n ; h\right)}\right), t \in[k \Delta t,(k+1) \Delta t), k=1, \ldots, n, \\
S_{T}^{\left(\alpha_{\Delta t}, n ; h\right)}=S_{0} \exp \left(v T+\sigma \mathbb{B}_{T}^{\left(\alpha_{\Delta t}, n\right)}+\gamma \mathbb{C}_{T}^{\left(\alpha_{\Delta t}, n ; h\right)}\right)
\end{array}\right\},
$$

where $v \in R, \sigma \in R \backslash\{0\}$ and $\gamma \in R$ are parameters determining the dynamics of the stock price as a function of the index dynamics. The discrete dynamics of the stock log-return is given by

$$
r_{k \Delta t}^{\left(\alpha_{\Delta t}, n ; h\right)}=\ln \left(\frac{S_{k \Delta t}^{\left(\alpha_{\Delta t}, n ; h\right)}}{S_{(k-1) \Delta t}^{\left(\alpha_{\Delta t}, n ; h\right)}}\right)=v \Delta t+\sigma \sqrt{\Delta t} M_{k}^{\left(\alpha_{\Delta t}\right)}+\gamma \sqrt{\Delta t} M_{k}^{\left(\alpha_{\Delta t}\right)} h\left(\sum_{i=1}^{k-1} \sqrt{\Delta t} M_{i}^{\left(\alpha_{\Delta t}\right)}\right), k=1, \ldots, n .
$$

Thus, if $\gamma \neq 0$, the stock log-return $r_{k \Delta t}^{\left(\alpha_{\Delta t}, n ; h\right)}$ depends on the entire path $\left\{M_{i}^{\left(\alpha_{\Delta t}\right)}, i=1, \ldots, k\right\}$. In the pre-limiting case, $\mathbb{S}_{[0, T]}^{\left(\alpha_{\Delta t}, n ; h\right)}$ approaches $\mathbb{S}_{[0, T]}^{\left(\alpha_{\Delta t} ; h\right)}=\left\{S_{0} \exp \left(v t+\sigma B_{t}^{\left(\alpha_{\Delta t}\right)}+\gamma C_{t}^{\left(\alpha_{\Delta t} ; h\right)}\right), t \in[0, T]\right\}$. In the limit $n \uparrow \infty, \mathbb{S}_{[0, T]}^{(\alpha \Delta, n ; h)}$ converges weakly in $\mathcal{D}[0, T]$ to $\mathbb{S}_{[0, T]}^{(h)}=\left\{S_{0} \exp \left(v t+\sigma B_{t}+\gamma C_{t}^{(h)}\right), t \in[0, T]\right\}$.

\footnotetext{
${ }^{44} \mathrm{See} / \mathrm{Hu}$ et al. $(2020 \mathrm{~b})$ for an application of the CSYIP using BM.

${ }^{45}$ See Cherny et al. (2003).
} 
To demonstrate the application of the model (35), (36), we use (36) to estimate the MSFT log-return $r_{k \Delta t}^{(n, \mathrm{MSFT})}$ over the period $\tau_{2}$. To capture likely heavy-tailed behavior, we identify $h(\cdot)$ as the Student's $t$ probability density function with $\kappa$ degrees of freedom. For convenience, we utilize the optimal path $M_{k}^{\left(\bar{\alpha}^{(F)} \text {,exo) }\right.}$ determined from the exogenous procedure in section 5.1 for the required path in (36). To establish the model parameters, $v, \sigma, \gamma$, and $\kappa$, we construct the conditional least squares minimization problem,

$$
\min _{\nu \in R, \sigma \in R, \gamma \in R, \kappa \in[5,30]}\left\|r_{k \Delta t}^{(n, \mathrm{MSFT})}-r_{k \Delta t}^{\left(\alpha_{\Delta t}, n ; h\right)}\right\|_{2}^{2} .
$$

Table 3 provides the resulting parameter estimates. The model (35), (36) with these parameter estimates will be used further in section 6.1.

\begin{tabular}{ccccc}
\hline$v$ & $\sigma$ & $\gamma$ & $\kappa$ & $\mathrm{RMSE}$ \\
0.272 & $3.44 \cdot 10^{-5}$ & $4.22 \cdot 10^{-2}$ & 6.24 & $1.86 \cdot 10^{-2}$ \\
\hline
\end{tabular}

Table 3: Parameters estimates obtained from (37)

\section{GJR option pricing}

Given the filtration $\mathbb{F}^{\left(n ; \mathbb{M}^{(\alpha \Delta t)}\right)}=\left\{\mathcal{F}_{k}^{\left(n ; \mathbb{M}^{(\alpha \Delta t)}\right.}=\sigma\left(M_{j}^{\left(\alpha_{\Delta t}\right)} ; j=1, \ldots, k\right), \quad \mathcal{F}_{k}^{\left(n ; \mathbb{M}^{(\alpha \Delta t)}\right)}=\{\varnothing, \Omega\}, k=\right.$ $1, \ldots, n\}$, consider the $\mathbb{F}^{\left(n ; \mathbb{M}^{(\alpha \Delta t)}\right)}$-adapted path-dependent GJR pricing tree determined by (36),

$$
\begin{aligned}
S_{k \Delta t}^{\left(\alpha_{\Delta t}, n ; h\right)}=S_{(k-1) \Delta t}^{\left(\alpha_{\Delta t}, n ; h\right)} \exp \left[v \Delta t+\sigma \sqrt{\Delta t}\left(M_{k}^{\left(\alpha_{\Delta t}\right)}-\right.\right. & \left.M_{k-1}^{\left(\alpha_{\Delta t}\right)}\right) \\
& \left.+\gamma \sqrt{\Delta t}\left(M_{k}^{\left(\alpha_{\Delta t}\right)}-M_{k-1}^{\left(\alpha_{\Delta t}\right)}\right) h\left(\sqrt{\Delta t} M_{k-1}^{\left(\alpha_{\Delta t}\right)}\right)\right),
\end{aligned}
$$

where $k=1, \ldots, n, n \Delta t=T$. Let $h(x) \geq 0, x \in R$, be a chosen probability density function 46 Define

$$
\eta_{k \Delta t}=\sigma+\gamma h\left(\sqrt{\Delta t} M_{k-1}^{\left(\alpha_{\Delta t}\right)}\right), k=1, \ldots, n ; \quad \eta_{0}=\sigma .
$$

Note that $\eta_{k \Delta t}^{(\alpha \Delta t)}$ represents the stock's time-varying factor-volatility at $k \Delta t$ as a function of the path of factor up and down movements $M_{k-1}^{\left(\alpha_{\Delta t}\right)}$. From (38), conditionally on $\mathcal{F}_{k}^{\left(n ; \mathbb{M}^{\left(\alpha_{\Delta t}\right)}\right)}, k=0, \ldots, n-1$,

$$
S_{(k+1) \Delta t}^{\left(\alpha_{\Delta t}, n ; h\right)}= \begin{cases}S_{(k+1) \Delta t}^{\left(\alpha_{\Delta t}, n, u ; h\right)}=S_{k \Delta t}^{\left(\alpha_{\Delta t}, n ; h\right)} \exp \left(v \Delta t+\eta_{k \Delta t}^{\left(\alpha_{\Delta t}\right)} \sqrt{\Delta t}\right), \text { w.p. } \mathbb{P}\left(M_{k+1}^{(\alpha)}=M_{k}^{(\alpha)}+1 \mid M_{k}^{(\alpha)}\right), \\ S_{(k+1) \Delta t}^{\left(\alpha_{\Delta t}, n, j ; h\right)}=S_{k \Delta t}^{\left(\alpha_{\Delta t}, n ; h\right)} \exp \left(v \Delta t-\eta_{k \Delta t}^{\left(\alpha_{\Delta t}\right)} \sqrt{\Delta t}\right), \text { w.p. } \mathbb{P}\left(M_{k+1}^{(\alpha)}=M_{k}^{(\alpha)}-1 \mid M_{k}^{(\alpha)}\right) .\end{cases}
$$

For $k=0,1, \ldots, n-1$, consider the replicating risk-neutral portfolio $P_{k \Delta t}^{\left(\alpha_{\Delta t}, n ; h\right)}=S_{k \Delta t}^{\left(\alpha_{\Delta t}, n ; h\right)} D_{k \Delta t}^{\left(\alpha_{\Delta t}, n ; h\right)}$ $f_{k \Delta t}^{\left(\alpha_{\Delta t}, n ; h\right)}$ with $P_{(k+1) \Delta t}^{\left(\alpha_{\Delta t}, n ; h\right)}=S_{(k+1) \Delta t}^{\left(\alpha_{\Delta t}, n ; h\right)} D_{k \Delta t}^{\left(\alpha_{\Delta t}, n ; h\right)}-f_{(k+1) \Delta t}^{\left(\alpha_{\Delta t}, n ; h\right)}$, given $\left.\mathcal{F}_{k}^{\left(n ; \mathbb{M}^{\mathbb{M}}\left(\alpha_{t}\right)\right.}\right)$. Thus, conditionally on $\mathcal{F}_{k}^{\left(n ; \mathbb{M}^{\left(\alpha_{\Delta}\right)}\right)}$,

$$
D_{k \Delta t}^{\left(\alpha_{\Delta t}, n ; h\right)}=\frac{f_{(k+1) \Delta t}^{(\alpha \Delta, n), h)}-f_{(k+1) \Delta t}^{(\alpha \Delta, n), h}}{e^{v \Delta t} S_{k \Delta t}^{\left(\alpha_{\Delta t}, n ; h\right)}\left(\exp \left(\eta_{k \Delta t}^{(\alpha \Delta t)} \sqrt{\Delta t}\right)-\exp \left(-\eta_{k \Delta t}^{\left(\alpha_{\Delta t}\right)} \sqrt{\Delta t}\right)\right)} .
$$

As portfolio $P_{(k+1) \Delta t}^{\left(\alpha_{\Delta t}, n ; h\right)}$ is now riskless, it follows that $P_{k \Delta t}^{\left(\alpha_{\Delta t}, n ; h\right)}=e^{-r_{f} \Delta t} P_{(k+1) \Delta t}^{\left(\alpha_{\Delta t}, n, u ; h\right)}$ with $P_{(k+1) \Delta t}^{\left(\alpha_{\Delta t}, n, u ; h\right)}=$ $D_{k \Delta t}^{\left(\alpha_{\Delta t}, n ; h\right)} S_{k \Delta t}^{\left(\alpha_{\Delta t}, n ; h\right)} \exp \left(v \Delta t+\eta_{k \Delta t}^{\left(\alpha_{\Delta t}\right)} \sqrt{\Delta t}\right)-f_{(k+1) \Delta t}^{(n, u)}$. Thus, conditionally on $\mathcal{F}_{k}^{\left(n ; \mathbb{M}^{\left(\alpha_{\Delta t}\right)}\right)}$

$$
f_{k \Delta t}^{\left(\alpha_{\Delta t}, n ; h\right)}=e^{-r_{f} \Delta t}\left(q_{(k+1) \Delta t}^{\left(\alpha_{\Delta t}, n ; h\right)} f_{(k+1) \Delta t}^{\left(\alpha_{\Delta t}, n, u ; h\right)}+\left(1-q_{(k+1) \Delta t}^{\left(\alpha_{\Delta t}, n ; h\right)}\right) f_{(k+1) \Delta t}^{\left(\alpha_{\Delta t}, n, d ; h\right)}\right),
$$

\footnotetext{
${ }^{46}$ For example, $h$ is the Student's $t$ density function with $\kappa$-degrees of freedom.
} 
where the risk-neutral probability, $q_{(k+1) \Delta t}^{(\alpha \Delta, n ; h)}$, for the time period $[k \Delta t,(k+1) \Delta t)$ is given by

$$
q_{(k+1) \Delta t}^{\left(\alpha_{\Delta t}, n ; h\right)}=\frac{\exp \left(r_{f} \Delta t-v \Delta t\right)-\exp \left(-\eta_{k \Delta t}^{\left(\alpha_{\Delta t}\right)} \sqrt{\Delta t}\right)}{\exp \left(\eta_{k \Delta t}^{\left(\alpha_{\Delta t}\right)} \sqrt{\Delta t}\right)-\exp \left(-\eta_{k \Delta t}^{\left(\alpha_{\Delta t}\right)} \sqrt{\Delta t}\right)}
$$

To leading order in $\Delta t$ we have

$$
q_{(k+1) \Delta t}^{\left(\alpha_{\Delta t}, n ; h\right)}=\frac{1}{2}+\frac{r_{f}-v-\left(\eta_{k \Delta t}^{\left(\alpha_{\Delta t}\right)}\right)^{2} / 2}{2 \eta_{k \Delta t}^{\left(\alpha_{\Delta t}\right)}} \sqrt{\Delta t} .
$$

Note that, conditionally on $\mathcal{F}_{k}^{\left(n ; \mathbb{M}^{(\alpha \Delta t)}\right)}$, the risk-neutral probability $q_{(k+1) \Delta t}^{(\alpha \Delta t, n ; h)}$ depends on the entire path $M_{k}^{(\alpha \Delta t)}$.

\subsection{Implied volatility}

Using the daily closing prices and the corresponding log-returns for MSFT, we apply the full GJR model to generate a binomial tree to construct call option prices using (40) and (41). CBOE option price data 47 , with all available strike values and expiration dates prior to $1 / 17 / 2020$, were collected for the date $7 / 1 / 2019$ for the underlying stock MSFT. The time series $\left\{\eta_{k \Delta t}^{\left(\alpha_{\Delta t}\right)}, k=1, \ldots, n\right\}$ (39) is based on the optimal trajectory $\left\{M_{k}^{\left(\bar{\alpha}^{(F)} \text {,exo }\right)}, k=1, \ldots, n\right\}$ obtained in section 5.1. The coefficients $v, \eta_{0}=\sigma, \gamma$, and the degrees of freedom $\kappa$ for the Student-t distribution used as the model for $h(\cdot)$, are taken from Table 3 .

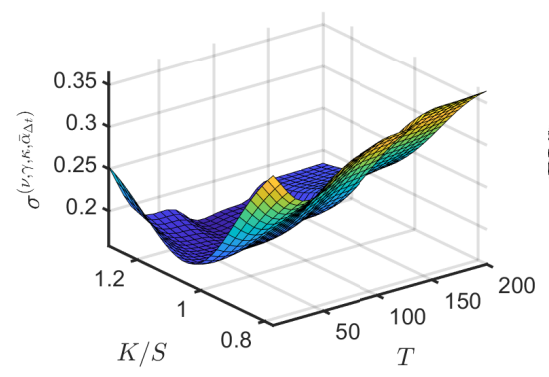

(a)

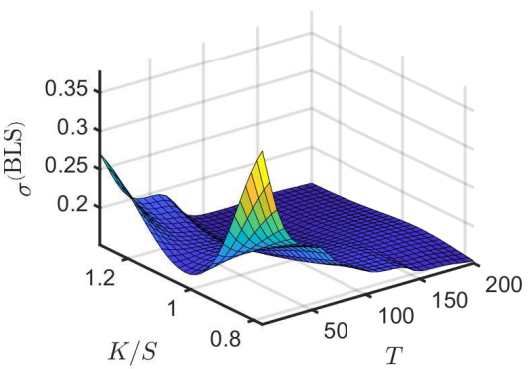

(b)

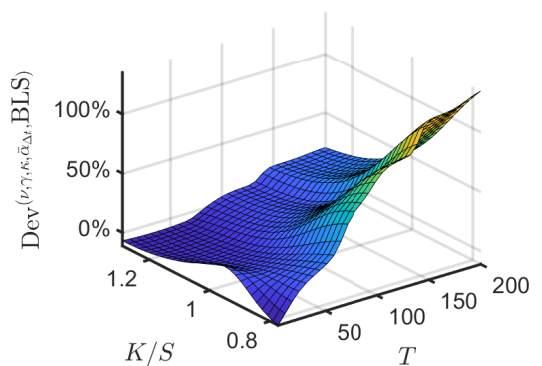

(c)

Figure 12: (a) The implied $\sigma^{\left(\nu, \gamma, \kappa, \bar{\alpha}_{\Delta t}\right)}$ surface generated by the path-dependent GJR pricing tree. (b) The implied $\sigma^{\text {(BLS) }}$ surface using the Black-Scholes formula for implied volatility. (c) The percent deviation surface $\operatorname{Dev}^{(\nu, \gamma, \kappa, \bar{\alpha} \Delta t, \mathrm{BLS})}$. Each surface is plotted as functions of moneyness $K / S$ and time to maturity $T$ (in days).

As in the numerical examples in sections 3.1 and 4.1, we compute the implied volatility, $\left.\sigma^{(\nu, \gamma, \kappa, \bar{\alpha}} \Delta t\right)$, based on the path-dependent GJR pricing tree. We also estimate the Black-Scholes implied volatility $\sigma^{(\mathrm{BLS})}$ surface for the same option prices, and define the percent deviation between the two surfaces, $\operatorname{Dev}^{(\nu, \gamma, \kappa, \bar{\alpha} \Delta t, \mathrm{BLS})}=$ $100\left(\sigma^{(\nu, \gamma, \kappa, \bar{\alpha} \Delta t)}-\sigma^{(\mathrm{BLS})}\right) / \sigma^{(\mathrm{BLS})}$. Fig. 12 shows the implied volatility surfaces plotted as functions of time to maturity $T$ (in days) and moneyness $K / S$. For maturity times $\lesssim 50$ days, both the GJR and the Black-Scholes surfaces contain volatility smiles and the deviation between the two surfaces is relatively small. Beyond 50 days, the Black-Scholes implied volatility surface flattens, while the GJR surface flattens only in the "out-of-the-money" region, leading to large differences between the two over the "in-the-money" region.

\footnotetext{
${ }^{47}$ See https://datashop.cboe.com/options-intervals
} 


\section{Conclusions}

In the option pricing literature since 1990, academic work on continuous-time option pricing models (CPMs) has greatly overshadowed the work on discrete-time option pricing models (DPMs). Moreover, with very few exceptions, DPMs are used only to approximate the CPMs (presumably to decrease the numerical complexity in generating Monte Carlo price trajectories). Those DPMs are placed directly into the riskneutral world and do not address an issue that is very important for every option trader: "Which DPM in the natural world is uniquely determined by the DPM introduced directly in the risk-neutral world?" The reason for the "subordinated" role of the DPM's is the "obsessive love" of academics for CPMs, arising from the alluring mathematical beauty of the semimartingale theory 48 of the Strasbourg school and the truly paramount Fundamental Theorem of Asset Pricing 49 However, crucial information found in DPMs in the natural world, such as the mean-return parameter, the probability for stock-upturn, and the distributional skewness and kurtosis, is lost in CPMs. Most notably, in CPMs the mean-return parameter is lost due to the assumed ability of the hedger to trade continuously in time. DPMs with defined dynamics in the natural world do not have this issue 50 That observation was the main motivation for this paper. Specifically, we have extended the classical Jarrow-Rudd pricing tree to include skewness and kurtosis in the underlying asset's return distribution in both the natural and in the risk-neutral world. We have extended Merton's option pricing tree model with hedging-transaction costs to our new generalized Jarrow Rudd (GJR) option pricing tree model. We have applied the Cherny-Shiryaev-Yor invariance principle and the Fama-French fivefactor model to further extend the GJR pricing tree model to cover path-dependent options. Our numerical examples include estimation of the implied surfaces of all parameters in the GJR pricing trees.

\section{References}

Atar, R. and Budhiraja, A. (2015). On the multi-dimensional skew Brownian motion. Stochastic Processes and their Applications, 125:1911-1925.

Bhat, H. and Kumar, N. (2012). Option pricing under a normal mixture distribution derived from the Narkov tree model. European Journal of Operational Research, 223:762-774.

Billingsley, P. (1999). Convergence of Probability Measures, Second Edition. Wiley-Interscience, New York.

Black, F. (1975). Fact and fantasy in the use of options. Financial Analysts Journal, 31(4):36-41.

Black, F. and Scholes, M. (1973). The pricing of options and corporate liabilities. Journal of Political Economy, 81:637-654.

Boyle, P. and Vorst, T. (1992). Optimal replication in discrete time with transaction costs. Journal of Finance, 47(4):271-293.

Brenner, M. and Galai, D. (1984). On measuring the risk of common stocks implied by options prices: A note. Journal of Financial and Quantitative Analysis, 19:403-412.

Broadie, M., Cvitanic, J., and Soner, H. (1998). Optimal replication of contingent claims under portfolio constraints. Review of Financial Studies, 11:59-79.

Chen, G. Y., Palmer, K., and Sheu, Y. C. (2008). The least cost super replicating portfolio in the Boyle-Vorst model with transaction costs. International Journal of Theoretical and Applied Finance, 11:55-85.

Cherny, A., Shiryaev, A., and Yor, M. (2003). Limit behavior of the "horizontal-vertical" random walk and some extensions of the Donsker-Prokhorov invariance principle. Theory of Probability and its Applications, $47(3): 377-394$.

\footnotetext{
${ }^{48}$ See Émery and Yor (2002), Jacod and Shirvaev (1987), and Protter (2004).

${ }^{49}$ Delbaen and Schachermayer (1994, 1998).

${ }^{50}$ See Kim et al. $(2016,2019)$, Hu et al. $(2020 a, b)$.
} 
Corns, T. R. A. and Satchell, S. E. (2007). Skew Brownian motion and pricing European options. European Journal of Finance, 13:523-544.

Cox, J., Ross, S., and Rubinstein, M. (1979). Options pricing: a simplified approach. Journal of Financial Economics, 7:229-263.

Davis, M., Panas, V., and Zariphopoulou, T. (1993). European option pricing with transaction costs. SIAM Journal Control and Optimization, 31:470-493.

Davydov, Y. and Rotar, V. (2008). On a non-classical invariance principle. Statistics \& Probability Letters, 78:2031-2038.

Delbaen, F., Kabanov, Y., and Valkeila, E. (2002). Hedging under transaction costs in currency markets: A discrete-time model. Mathematical Finance, 12:45-61.

Delbaen, F. and Schachermayer, W. (1994). A general version of the fundamental theorem of asset pricing. Mathematische Annalen, 300:463-520.

Delbaen, F. and Schachermayer, W. (1998). The fundamental theorem of asset pricing for unbounded stochastic processes. Mathematische Annalen, 312:215-250.

Donsker, M. (1951). An invariant principle for certain probability limit theorems. Memoirs of the American Mathematical Society, 6:1-10.

Duan, J. and Simonato, J. (2001). American option pricing under GARCH by a Markov chain approximation. Journal of Economics and Dynamic Control, 25:1689-1718.

Duffie, D. (2001). Dynamic Asset Pricing Theory, Third Edition. Princeton University Press.

Edirisinghe, C., Naik, V., and Uppal, R. (1993). Optimal replication of options with transactions costs and trading restrictions. Journal of Financial and Quantitative Analysis, 28:117-138.

Émery, M. and Yor, M. (2002). Séminaire de Probabilités 1967-1980: A Selection in Martingale Theory (Eds.). Springer, Berlin.

Fama, E. and French, K. (2015). A five-factor asset pricing model. Journal of Financial Economics, 116:1-22.

Fan, K., Shen, Y., Siu, T.-K., and Wang, R. (2016). On a Markov chain approximation method for option pricing with regime switching. Journal of Industrial and Management Optimization, 12:529-541.

Fuh, C.-D., Ho, K., Hu, I., and Wang, R.-H. (2012). Option pricing with Markov switching. Journal of Data Science, 10:483-509.

Gikhman, I. and Skorokhod, A. (1969). Introduction to the Theory of Random Processes. W.B. Saunders Company.

Guasoni, P., Lépinette, E., and Rásonyi, M. (2012). The fundamental theorem of asset pricing under transaction costs. Finance and Stochastics, 16:741-777.

Harrison, J. and Shepp, L. (1981). On skew Brownian motion. The Annals of Probability, 9:309-313.

Heston, S., Loewenstein, M., and Willard, G. (2007). Options and bubbles. Review of Financial Studies, 20:359-390.

Hilber, N., Reich, N., Schwab, C., and Winter, C. (2009). Numerical methods for Lévy processes. Review of Financial Studies, 13:471-500.

Hodges, S. and Neuberger, A. (1989). Optimal replication of contingent claims under transactions costs. Review of Futures Markets, 8:222-239.

Holland, P. W. and Welsch, R. E. (2007). Robust regression using iteratively reweighted least-squares. Communications in Statistics - Theory and Methods, 6(1977):813-827. 
Hu, Y., Shirvani, A., Stoyanov, S., Kim, Y. S., Fabozzi, F. J., and Rachev, S. T. (2020a). Option pricing in markets with informed traders. International Journal of Theoretical and Applied Finance, 23(6):2050037.

Hu, Y., Shirvani, A., Lindquist, W. B., Fabozzi, F. J., and Rachev, S. T. (2020b). Option pricing incorporating factor dynamics in complete markets. Journal of Risk and Financial Management, 13(12):321.

Hull, J. (2012). Options, Futures, and Other Derivatives, Eighth Edition. Pearson.

Hurst, S., Platen, E., and Rachev, S. (1999). Option pricing for a logstable asset price model. Mathematical and Computer Modelling, 29:105-119.

Itô, K. and McKean, H. (1996). Diffusion Processes and Their Sample Paths. Springer, Berlin.

Jacod, J. and Shiryaev, A. N. (1987). Limit Theorems for Stochastic Processes. Springer-Verlag Berlin and Heidelberg GmbH \& Co. KG.

Jarrow, R., Protter, P., and Shimbo, K. (2010). Asset price bubbles in incomplete markets. Mathematical Finance: An International Journal of Mathematics, Statistics and Financial Economics 20, 20(2):145-185.

Jarrow, R. and Rudd, A. (2012). Option Pricing, Irwin. Homewood, IL.

Kabanov, Y. and Safarian, M. (1997). On Icland's strategy of option pricing with transaction costs. Finance and Stochastics, 1:239-250.

Kabanov, Y. and Stricker, C. (2001). The Harrison-Pliska arbitrage pricing theorem under transaction costs. Journal of Mathematical Economics, 35:185-196.

Karandikar, R. and Rachev, S. (1995). A generalized binomial model and option pricing formulae for subordinated stock-price processes. Probability and Mathematical Statistics, 15:417-447.

Kijima, M. and Yoshida, M. (1993). A simple option pricing model with Markovian volatilities. Journal of the Operations Research Society of Japan, 36:149-166.

Kim, Y., Stoyanov, S., Rachev, S., and Fabozzi, F. (2016). Multi-purpose binomial model: fitting all moments to the underlying Brownian motion. Economics Letters, 145:225-229.

Kim, Y., Stoyanov, S., Rachev, S., and Fabozzi, F. (2019). Enhancing binomial and trinomial option pricing models. Finance Research Letters, 28:185-190.

Knez, P. and Ready, M. (1997). On the robustness of size and book-to-market in cross-sectional. Journal of Finance, 52:1355-1382.

Lai, T. and Lim, T. (2009). Option hedging theory under transaction costs. Journal of Economic Dynamics E Control, 33:1945-1961.

Lang, R. (1995). Effective conductivity and skew Brownian motion. Journal of Statistical Physics, $80: 125-146$.

Lejay, A. (2006). On the constructions of the skew Brownian motion. Probability Surveys, 3:413-466.

Leland, H. (1985). Option pricing and replication with transactions costs. Journal of Finance, 40:283-1301.

Li, L. (2019). On general skew Brownian motions. preprint: https://arxiv.org/abs/1812.08415.

Melick, R. and Thomas, C. (1997). Recovering an asset's implied pdf from option prices: an application to crude oil during the Gulf Crisis. Journal of Financial and Quantitative Analysis, 32:91-115.

Melnikov, A. and Petrachenko, Y. (2005). On option pricing in binomial market with transaction costs. Finance and Stochastics, 9:141-149.

Merton, R. (1973). Theory of rational option pricing. Bell Journal of Economics and Management Science, $4: 141-183$. 
Merton, R. C. (1990). Continuous-Time Finance. Blackwell Publishing, Oxford, UK.

Palmer, K. (2001). A note on the Boyle-Vorst discrete-time option pricing model with transactions costs. Mathematical Finance, 11:357-363.

Pasquariello, P. (2014). Financial market dislocations. The Review of Financial Studies, 27:1868-1914.

Pregibon, D. (1981). Logistic regression diagnostics. Annals of Statistics, 9(4):705-724.

Prokhorov, Y. (1956). Convergence of random processes and limit theorems in probability theory. Theory of Probability and its Applications, 1:157-214.

Protter, P. (2004). Stochastic Integration and Differential Equations, Second Edition. Springer, Berlin.

Ramirez, J. (2011). Multi-skewed Brownian motion and diffusion in layered media. Proceedings of the American Mathematical Society, 139:3739-3752.

Revuz, D. and Yor, M. (1994). Continuous Martingales and Brownian Motion. Springer-Verlag, Berlin.

Ross, S. (2015). The recovery theorem. Journal of Finance, 70:615-648.

Simonato, J. (2011). Computing American option prices in the lognormal jump-diffusion framework with a Markov chain. Finance Research Letters, 8:220-226.

Skorokhod, A. V. (2005). Basic Principles and Applications of Probability Theory. Springer.

Stettner, L. (1997). Option pricing in the CRR model with proportional transaction costs: A cone transformation approach. Applicationes Mathematicae, 4:475-514.

Steutel, F. and van Harn, K. (2004). Infinitely Divisibility of Probability Distributions on the Real Line. Marcel Dekker, New York.

Trutnau, G., Ouknine, Y., and Russo, F. (2015). On countably skewed Brownian motion with accumulation point. Electronic Journal of Probability, 20(82):1-27.

Van, d. H. J. and Elliott, R. (2012a). American option prices in a Markov chain market model. Applied Stochastic Methods in Business and Industry, 28:35-59.

Van, d. H. J. and Elliott, R. (2012b). Asset pricing using finite state Markov chain stochastic discount functions. Stochastic Analysis and Applications, 30:865-894.

Vogel, H. (2018). Financial Market Bubbles and Crashes, Second Edition. Palgrave Macmillan, Cham, Switzerland.

Yamada, Y. and Primbs, J. (2004). Properties of multinomial lattices with cumulants for option pricing and hedging. Asia-Pacific Financial Markets, Springer; Japanese Association of Financial Economics and Engineering, 11(3):335-365. 